\title{
THAP1 Modulates Oligodendrocyte Maturation by Regulating ECM Degradation in Lysosomes
}

Dhananjay Yellajoshyula ${ }^{*}$, Samuel S. Pappas ${ }^{4,5}$, Abigail Rogers ${ }^{1}$, Biswa Choudhury ${ }^{6}$, Xylena Reed ${ }^{2}$, Jinhui Ding', Mark R. Cookson², Vikram Shakkottai', Roman Giger ${ }^{3}$ and William T. Dauer $4,5,7^{*}$

1Department of Neurology, University of Michigan, Ann Arbor, MI, USA

${ }^{2}$ Cell Biology and Gene Expression Section, Laboratory of Neurogenetics, National Institute of Aging, National Institutes of Health, 9000 Rockville Pike, Bethesda, MD, USA.

3Department of Cellular \& Developmental Biology, University of Michigan, Ann Arbor, MI, USA

${ }^{6}$ Department of Cellular and Molecular Medicine, UCSD, La Jolla, California, USA

4Peter O'Donnell Jr. Brain Institute, ${ }^{5}$ Department of Neurology, ${ }^{7 D e p a r t m e n t}$ of Neuroscience, University of Texas Southwestern Medical Center, Dallas, Texas, USA.

*Corresponding authors - william.Dauer@UTSouthwestern.edu;

dyellaj@med.umich.edu

\section{ABSTRACT}

Mechanisms controlling myelination during CNS maturation play a pivotal role in the development and refinement of CNS circuits. The transcription factor THAP1 is essential for timing the inception of myelination during CNS maturation through a cellautonomous role in the oligodendrocyte lineage. Here, we demonstrate that THAP1 modulates ECM composition by regulating glycosaminoglycan (GAG) catabolism within oligodendrocyte progenitor cells (OPCs). Thap 1\%OPCs accumulate and secrete excess GAGs, inhibiting their maturation through an auto-inhibitory mechanism. THAP1 controls GAG metabolism by binding to and regulating the GusB gene encoding $\beta$ glucuronidase, a GAG-catabolic lysosomal enzyme. Applying GAG-degrading enzymes or overexpressing $\beta$-glucuronidase rescues Thap 1\%OL maturation deficits in vitro and in vivo. Our studies establish lysosomal GAG catabolism within OPCs as a critical mechanism regulating oligodendrocyte development. 


\section{INTRODUCTION}

The oligodendrocyte (OL) lineage plays a critical role in CNS development and function (Almeida and Lyons, 2017; Bergles and Richardson, 2015). The OL lineage in its mature cell state is responsible for wrapping axons with an insulating myelin sheath that supports rapid neurotransmission (Waxman and Bennett, 1972). Developmental myelination during a critical period of post-natal development plays an important role in the establishment of neural circuits (Fields, 2008; Rice and Barone, 2000; Wang et al., 2018). Regulated myelination in the adult CNS is also increasingly recognized to play an essential role in circuit plasticity, including motor learning (Bacmeister et al., 2020; Bergles and Richardson, 2015; McKenzie et al., 2014; Pepper et al., 2018; Xiao et al., 2016). Mechanisms regulating OL maturation are therefore central to the development of neuronal circuits as well as their plasticity and maintenance.

OL differentiation leading to myelination is regulated by cell-intrinsic pathways (Elbaz and Popko, 2019) and extrinsic signals that emanate from axons and the surrounding extracellular matrix (ECM) (Colognato and Tzvetanova, 2011; Harlow and Macklin, 2014; Sandvig et al., 2004). The ECM is a complex three dimensional structure surrounding cells, providing both a physical scaffold and a growth factor organizing center orchestrating signaling cascades that regulate mobility, development and function (Colognato and Tzvetanova, 2011; Fawcett et al., 2019; Theocharidis et al., 2014). The ECM is composed of glycosaminoglycans (GAGs), a class of long unbranched mucopolysaccharides, GAG-modified proteoglycans (GAG-PG) and fibrous proteins (e.g., collagen, elastin) (Esko et al., 2009). Considerable literature demonstrates that GAGs in their free form and as proteoglycans (e.g., chondroitin sulfate proteoglycan; CSPG) inhibit $\mathrm{OL}$ maturation both in vitro and in vivo, demonstrating that GAGs powerfully regulate OL development (Karus et al., 2016; Keough et al., 2016; Kuboyama et al., 2017; Lau et al., 2012; Pu et al., 2018; Siebert and Osterhout, 2011; Sloane et al., 2010).

GAG and proteoglycan content is determined through a balance of synthesis, secretion, and catabolism (Esko et al., 2009; Nowicka and Greda, 2019; Song and Dityatev, 2018). In addition to the extracellular degradation of ECM components by secreted proteases (Berezin et al., 2014), receptor and cell surface proteoglycans and GAGs are endocytosed and enzymatically catabolized in lysosomes (Rainero, 2016; Rappaport et al., 2016; Winchester, 2005). Failure of intracellular GAG catabolism results in mucopolysaccharidoses, a class of lysosomal storage disorders (Fiorenza et al., 2018; Kobayashi, 2019; Winchester, 2005). Despite their importance, the sources and cellular mechanisms regulating GAG content and composition in the CNS are ill defined. Most studies on the cellular origins of ECM have focused on CSPG synthesis from astrocytes (Jones et al., 2003; Sandvig et al., 2004; Song and Dityatev, 2018). Study of ECM metabolism within the OL lineage itself has been limited (Courel et al., 1998; Garwood et al., 2004; Song and Dityatev, 2018; Yim et al., 1993), despite the likely importance of these processes for OL biology in normal development and disease states. 
We demonstrated a cell autonomous role for the transcription factor THAP1 in regulating the maturation of OPC to mature myelinating OLs (Yellajoshyula et al., 2017). Loss-of-function mutations in the THAP1 gene cause the neurodevelopmental disorder DYT6 dystonia, directly implicating these events in dystonia pathogenesis (Fuchs et al., 2009). Conditional deletion of Thap1 from the OL lineage significantly impairs CNS myelination by retarding the transition of OLs to mature myelin producing cells, without overtly altering the number of oligodendrocyte progenitor cells (OPC)(Yellajoshyula et al., 2017). The marked delay in myelination corresponds to the development of motor dysfunction in the Thap1 mutants. The mechanism whereby THAP1 regulates OL lineage maturation was not defined in this study.

Here, we identify a direct role for THAP1 in regulating GAG catabolism and demonstrate directly that dysregulation of GAG catabolism within OPCs impairs their maturation. Thap1\% OPCs auto-inhibit their own maturation by accumulating and secreting excess GAGs. LC/MS analyses of OPC conditioned media demonstrate they predominantly secrete chondroitin 4-sulfate, which is significantly increased in Thap 1\% cells. THAP1 regulates GAG catabolism by binding to and regulating the GusB (MPS7) gene encoding the lysosomal GAG-catabolizing enzyme $\beta$-glucuronidase. Treatment with GAG degrading enzymes or overexpressing $\beta$-glucuronidase rescues the Thap1\% OPC deficits in OL maturation and CNS myelination, establishing that excess GAGs mediate maturation delay in the Thap 1/- oligodendroglial lineage. These findings newly establish GAG catabolism as a major cell intrinsic regulatory pathway driving OL maturation.

\section{RESULTS}

THAP1 regulates ECM pathway composition during OL development.

We demonstrated previously that THAP1 plays a cell-autonomous role in regulating myelination during early postnatal CNS development (Yellajoshyula et al., 2017). To begin to examine the mechanisms responsible for this role, we performed RNAseq at multiple time points on cultured control (Thap1+/+) and Thap1\% (derived from Thap $1^{\text {flx } / \text {; }}$; Olig2-Cre+) OPC under differentiating conditions. Significantly fewer Thap1/- OPC matured to express myelin basic protein (MBP+) following 4 days in vitro (DIV4) of treatment with $40 \mu \mathrm{g} / \mathrm{ml}$ T3 thyroid hormone (Fig. 1A-B; Control (Thap1+/+) $=79.44 \% \pm$ 4.58; Thap1\% $=83.28 \% \pm 6.83$; t-test $\mathrm{p}<0.001)$. MBP+ Thap1\% OLs are also significantly smaller (Fig. 1A,D) and exhibit less intense MBP staining than controls (Fig. $1 A, C)$. We isolated total mRNA from progenitor (OPC) and differentiating OLs (DIV2 \& 4) from three clonal lines each for Thap1+/+ and Thap1-- genotypes (Fig. 1E). We used DESeq2 for the RNAseq data derived from 18 samples to identify THAP1-dependent differentially expressed genes (DEG), requiring a greater than $\log _{2} 1.5$ fold change and $p<0.05$ significance by the Benjamini-Hochberg false discovery procedure. These analyses identified 1132 (OPC), 703 (DIV2), and 1059 (DIV4) DEGs (Fig. 1G; Table S1).

Analyses of the RNAseq data using estimated sample distance (Heat map, Fig. S1A) and principal component analysis (Fig. S1B) demonstrated that, within the same genotype, differentiating OL (DIV2 \& 4) were distinctly different from OPC. Thus we focused on DIV2 \& 4 cells, identifying two groups of THAP1-dependent differentially 
expressed genes (DEG): those in immature (DIV2) cells, and those persisting through maturation (i.e., differentially expressed at both DIV2 \& 4) (Fig. 1F). There were 703 DEG limited to DIV2 and 277 DEG present at both DIV2 \& 4 (Fig. 1F). These DEG were used for enrichment analysis of GO (Gene Ontology) terms to identify overrepresented biological pathways using both GENEONTOLOGY (http://geneontology.org) and DAVID (https://david.ncifcrf.gov/home.jsp) (Table S2). GO enrichment analyses (see Methods) demonstrated significant overrepresentation of ECM-related pathways in both groups of genes (Fig. 1H, S1C \& Table S2). Specific pathways identified were (1) GAG metabolism; (2) ECM-binding (including members of integrin and collagen families); (3) signaling components (Rho GTPases); and (4) cytoskeletal polymerization \& filopodium assembly (Fig. 1H \& S1C). These analyses suggested strongly that ECM pathways are dysregulated in the Thap1\% oligodendroglial lineage.

\section{Accumulation of Glycosaminoglycan in Thap 1\% oligodendrocytes.}

Guided by the strong representation of ECM and particularly GAG metabolism pathways, we explored whether Thap1\%cultures exhibited abnormalities in the levels of GAGs. We focused on two species most closely linked to oligodendrocyte biology: chondroitin sulfate (CS) and hyaluronate (HA-GAG) (Karus et al., 2016; Siebert and Osterhout, 2011; Sloane et al., 2010). Immunostaining of non-permeabilized mature (DIV4) cultures demonstrated that Thap1\% cells exhibited significantly increased staining for CS- and HA-GAG (Fig. 2A-D) and prominent accumulation around smaller OL processes (Fig. S2A). Staining specificity was confirmed by treating the cultures with chondroitinase $\mathrm{ABC}(\mathrm{ChABC})$ or hyaluronidase, which removed the relevant signals (Fig. 2 A-D). Thap1\% cultures contained a greater number of positive cells as well as significantly greater intensity of staining per cell (Fig. 2 A-D). Measurement of bulk GAG content using the carbazole assay confirmed significantly increased GAGs in Thap1\% cultures (Fig. S2B; OPC; Thap $1^{++}=0.066 \mathrm{AU} \pm 0.0006$; Thap1-1- $=0.094 \mathrm{AU} \pm 0.0022$; t-test: $\mathrm{t}_{(2)}=12.70 ; \mathrm{p}=0.006$ \& OL - Thap1+/+ $=0.036 \mathrm{AU} \pm 0.0023 ;$ Thap1-- $=0.059 \mathrm{AU} \pm$ 0.0019 ; $t$-test: $\left.t_{(2)}=7.96 ; p=0.015\right)$. These findings are consistent with the RNA-Seq data indicating that THAP1 participates in a GAG metabolism-related pathway.

\section{Chondroitin-4-sulfate is the major GAG elevated in Thap1\% OL.}

Little is known regarding the contribution of the OL lineage to the ECM, such as its ability to produce and secrete GAGs or the identity of those produced. Several species of GAGs (CS, HS and HA) regulate CNS development, including OL lineage maturation (Harlow and Macklin, 2014; Karus et al., 2016; Properzi et al., 2008; Siebert and Osterhout, 2011; Sloane et al., 2010). To determine if GAG species accumulate within THAP1 mutant OL and to characterize their identity, we employed glycan reductive isotope labeling (GRIL) combined with liquid chromatography tandem mass spectrometry (LC/MS) to quantify CS, HS and HA GAGs (Lawrence et al., 2008) in cell homogenates (intracellular) and supernatant (secreted) from DIV4 Thap $1^{+/+}$and Thap $1^{-/-}$ cultures (schematic, Fig. 2E). These analyses demonstrated that there are striking changes in the levels of secreted GAGs during OL maturation (Fig. 2F \& S2C). Media from Thap1+/+ OPC cultures exhibited 8 -fold higher GAG levels than in mature OLs, over $95 \%$ of which is CS-GAG (Fig. 2F \& S2C). In contrast, while significant differences in the composition of GAG were observed in cell homogenates (Fig. 2I \& S2D), the 
relative differences were far less than those observed in the media. THAP1 depletion significantly increased both secreted and cellular GAG (CS, HS and HA combined) levels (Fig. 2G,J \& S2C-D). These differences were primarily driven by increases in CSGAG, the dominant GAG species observed (Fig. 2F \& S2C). Levels of HA- \& HS-GAGs were also significantly increased in Thap1\% cultures (Fig. S2C-D).

The CS-GAG family includes many species with differing amounts and patterns of sulfation that can be detected and resolved by LC/MS. In Thap1+/+ cultures, we found that the dominant form of CS-GAG secreted by OL cultures is chondroitin-4-sulfate ("C-4S"), constituting 85 - 90\% of CS-GAG secreted by OL cultures (Fig. 2H,K \& S3EF). Chondroitin-6-sulfate ("C-6S") was the only other abundant species ( 10\% of CSGAGs) with negligible amounts of non- and bi-sulfated species ("C-2,6S" \& "C-4,6S") (Fig. 2H,K \& S3E-F). Consistent with the immunostaining data (Figs. 2A-B), C-4S and C-6S were significantly increased in Thap 1\% cultures (Fig. $2 \mathrm{H}, \mathrm{K} \&$ S SE-F). We directly tested the ability of these forms of CS-GAG to alter oligodendroglial lineage progression by applying these species to differentiating (wild type) OPC cultures (Figs. 2L-N). C-4S and C-6S $(10 \mu \mathrm{g} / \mathrm{ml})$ each caused a dose dependent inhibition of OL maturation, impairing the progression to mature MBP+OLs and causing mature cells to occupy a smaller area (Fig. 2L-N). These data demonstrate that the specific species of CS-GAG elevated in Thap1-- cultures impair oligodendroglial maturation.

\section{Accumulation of secreted CS-GAG contributes to Thap1\% maturation deficits and} exerts an inhibitory paracrine effect.

To further explore the hypothesis that increased CS-GAG secretion is responsible for the maturation impairments of Thap1\%-cultures, we tested the ability of the CS-GAG degrading enzyme ChABC to rescue this phenotype. Consistent with our hypothesis, treatment of Thap 1\% cultures with $0.1 \mathrm{U} / \mathrm{ml}$ ChABC significantly increased the proportion of myelinating $\mathrm{OL}$, by increasing the number MBP+ cells ( 3-fold increase), the intensity of MBP staining ( 3-fold increase), and OL size ( 2-fold increase in cell surface area) (Fig. 3A-D). Since Thap1\% OLs secrete excess CS-GAGs (Fig. 2), we hypothesized that they may exert non-cell autonomous paracrine effects on neighboring cells. We tested this idea by exploring the ability of Thap1+/+ OPC (GFP-labeled with lentivirus; "LVGFP") to mature when co-cultured with unlabeled Thap1\% or Thap $1+/+$ OL (Figs. 3E \& S3; cultures contain 1 Thap1+/+:LV-GFP cell: 10 Thap1\% or Thap1+/+ OL). Thap1\% OLs significantly impaired the maturation of Thap1+/+;LV-GFP cells (Fig. $3 \mathrm{~F}-\mathrm{H}$ ). Compared to being cultured in the presence of Thap1+/+ OLs, Thap1+/+;LV-GFP OPCs cultured in the presence of Thap1\% OLs exhibited significantly lower MBP expression (Fig. 3 F-G; 4fold lower MBP intensity) and covered a significantly smaller area (Fig. 3 F,H; 30\% smaller area). This inhibitory paracrine effect was eliminated by the addition of ChABC to the media (Fig. $3 \mathrm{~F}-\mathrm{H}$ ). Considered together, these data are consistent with a model whereby Thap 1\% OLs inhibit the maturation of surrounding cells through a CS-GAG inhibitory paracrine signal.

THAP1 binds to and regulates the GusB (MPS VII) gene encoding the GAG degrading enzyme $\beta$-glucuronidase 
To mechanistically dissect the connection between THAP1 and GAG metabolism, we examined our RNAseq analyses (DIV2 and DIV2 \& 4 common; Fig. 1F \& Table S1). We identified a set of 42 genes (Table S3) relevant to GAG and proteoglycan metabolism that were differentially regulated in Thap 1\% cultures (Table S2). We focused our attention on genes that are (1) directly involved in GAG metabolism and (2) direct targets of THAP1. These criteria identified the Gusb gene encoding $\beta$-glucuronidase as a strong THAP1 candidate target. $\beta$-glucuronidase is an essential lysosomal enzyme that participates in the later steps of the catabolism of multiple species of GAG polysaccharides and proteoglycans. Recessively inherited loss-of-function mutations in GUSB causes the mucopolysaccharidosis Sly's syndrome (MPS VII) (Heuer et al., 2001; Kubaski et al., 2017; Kyle et al., 1990; Paigen, 1989; Ray et al., 1999; Zielonka et al., 2017). Prior ChIPseq data from human K562 cells demonstrates that THAP1 is bound to the GUSB promoter (Fig. 4A; ENCODE dataset). We confirmed that THAP1 plays a critical role in the transcription of the GusB locus in the OL lineage (Fig. 4B-E). Quantitative chromatin immunoprecipitation (qChIP) analyses in DIV4 OL demonstrated that THAP1 is bound to the GusB promoter in the OL lineage (Fig. 4B). Consistent with that finding, GusB expression is significantly and profoundly reduced in Thap 1/- cultures (OPC through DIV4; Fig. 4E). We previously demonstrated that most THAP1 targets are also bound by $Y Y 1$, a transcription factor known to regulate OL maturation, and that YY1 DNA binding is significantly reduced in Thap1-- OLs (Yellajoshyula et al., 2017). Analyses of the GusB locus demonstrate that it is bound by YY1 in a THAP1-dependent manner (Fig. 4C). H3K4me3, an epigenetic modification associated with active transcription, is significantly reduced at the GusB locus in Thap ${ }^{-/}$OL, further connecting THAP1 loss to GusB function (Fig. 4D).

Consistent with significantly reduced GusB expression in Thap1/- OL (Fig. 4E), we also find reduced $\beta$-glucuronidase enzymatic activity in cell culture and CNS tissue, as measured by fluorometry (MUG assay). In Thap1+/+ cultures, $\beta$-glucuronidase activity was comparable in OPC and OL (DIV4) (Fig. S4A-B). Thap1\% OLs exhibited a nearly 20 -fold decrease of $\beta$-glucuronidase activity (Fig. 4F-G). In Thap1-cortical tissue, GusB mRNA was decreased by $\sim 80 \%$ (Fig. $4 \mathrm{H}$ ) and $\beta$-glucuronidase activity was reduced by $\sim 75 \%$ (Fig. 4l-J). Several other brain regions assessed (cortex, striatum, corpus callosum and cerebellum) showed similar reductions in $\beta$-glucuronidase activity (Fig. $\mathrm{S} 4 \mathrm{C}$ ). $\beta$-Glucuronidase is a lysosomal enzyme, suggesting that loss of its activity should cause lysosomal accumulation of CS-GAGs. Indeed, assessing intracellular CS-GAG by staining permeabilized cells demonstrated significantly higher levels in Thap1/- OL cultures (Fig. 4K-M). Co-labeling cells for CS-GAG and the pan-lysosomal marker LAMP1 confirmed that the intracellular CS-GAG accumulation is almost exclusively localized to lysosomes (Fig. 4N, S4D). Considered together with the effects of THAP1 at the GusB locus, these data indicate a direct and essential role for THAP1 in regulating levels of CNS $\beta$-glucuronidase.

GUSB overexpression rescues THAP1 mutant GAG homeostatic and oligodendroglial maturation defect in vitro and in vivo

To formally test the necessity of reduced $\beta$-glucuronidase for THAP1-related GAG homeostatic and oligodendroglial lineage defects, we pursued rescue studies with a 
GUSB transgene (TgGUS; (Kyle et al., 1990)). Prior work demonstrated that the TgGUS does not produce a phenotype when in the hemizygous state on a wild type background (Kyle et al., 1990). We ingressed TgGUS onto the CNS conditional Thap1- background. We used these mice to derive OPCs from SVZ derived neural stem cells (as previously described (Yellajoshyula et al., 2017)) from Thap1+/+ and Thap1\% with or without the TgGUS transgene. We confirmed that the TgGUS transgene significantly increased $\beta$ glucuronidase activity in Thap1\% OPCs (Fig. 5A) and that Thap1\%-;gGus OPCs normally express the OPC-specific markers OLIG2 and CSPG4 (NG2) (Fig. 5B).

We began assessing the effects of increasing $\beta$-glucuronidase by measuring CS-GAG levels in Thap1\% OPCs. Immunostaining Thap1\%;TgGUS OL (+T3-DIV4) for CS-GAG under non-permeabilizing conditions demonstrated that the TgGUs transgene completely reversed the CS-GAG accumulation phenotype (Fig. 5C-D). At DIV4, Thap1-/;gGus cultures also exhibited a significantly greater number of myelinating OL (\%MBP/OL;Fig. 5E-F) and increased MBP staining (MBP+ intensity; Fig. 5E-F) compared to Thap1\% OL. Having confirmed in vitro rescue we next tested the ability of TgGUS transgene to rescue THAP1-related myelination defects in vivo. We confirmed that the TgGus allele increases $\beta$-glucuronidase activity in all CNS regions tested (Fig. S5). Using electron microscopy we assessed 1) density of myelinated axons; 2) myelin thickness; and 3) size of myelinated axons. We performed these analyses in P21 tissue from the genu of corpus callosum tissue from CNS conditional knockout Thap1 (nestin-cre; "N-CKO") or control (Thap1+/+) mice with or without the TgGUS transgene (Fig. 6 A-F). N-CKO;TgGUs mice exhibited a 2-fold increase in the density of myelinated axons compared to N-CKO mice without the transgene (Fig. 6 A-B). TgGUS did not fully restore myelinated axon density to control levels, however. The abnormally high g-ratio characteristic of $\mathrm{N}-\mathrm{CKO}$ mice, in contrast, was rescued completely by restoration of $\beta$-glucuronidase (Fig. 6 A,C-D,F). NCKO mice exhibit a modest but significant increase in the size of the myelinated axons, which was also rescued by the TgGUS transgene (Fig. 6 A,E). These findings establish $\beta$ glucuronidase activity as a key mediator of THAP1-related CNS pathology.

\section{DISCUSSION}

Our studies establish THAP1 as a transcriptional regulator of OL maturation via its control of GAG-metabolism. We demonstrate that THAP1 directly binds to and regulates the GusB gene encoding $\beta$-glucuronidase, a lysosomal GAG-degrading enzyme. THAP1 loss dramatically decreases GusB mRNA and $\beta$-glucuronidase enzymatic activity, causing CS-GAG lysosomal accumulation and increasing secretion. Multiple lines of evidence support CS-GAG accumulation as the key mechanism responsible for delaying maturation of the Thap1\% OL lineage. Preventing abnormal accumulation of GAGs rescues the Thap1\% OL maturation defect whereas administering GAG species that accumulate in Thap1\% cultures (C-4S, C-6S) impairs myelination of control OLs. Moreover, overexpressing GUSB rescues GAG accumulation and OL maturation, and ameliorates CNS myelination delay in Thap $1 \%$ mice. Considered together, our findings identify a novel cell autonomous mechanism of $\mathrm{OL}$ lineage regulation and provide insight into the molecular pathophysiology of THAP1 loss-of-function, the cause of DYT6 dystonia. 
The role of CS-GAGs (and CSPGs) in regulating OL differentiation has been well studied (Harlow and Macklin, 2014; Karus et al., 2016; Keough et al., 2016; Kuboyama et al., 2017; Lau et al., 2012; Pu et al., 2018; Siebert and Osterhout, 2011; Sloane et al., 2010). CSPGs have been demonstrated to inhibit OL differentiation through their interactions with the OPC resident receptor-type protein-tyrosine phosphatase (RPTP) subtypes, RPTP $\zeta$ and RPTPo, to suppress the activation of Rho-associated kinase (ROCK), which is antagonistic to OL differentiation (Kuboyama et al., 2016; Pendleton et al., 2013). Studies exploring the cellular source of these signals have largely focused on astrocytes (Asher et al., 2000; Feliu et al., 2020; Laabs et al., 2007; McKeon et al., 1999; Tang et al., 2003). Here we demonstrate that the OL lineage itself is a functionally important source of CS-GAGs that is auto-inhibitory to its development. Several prior and recent studies support our hypothesis that include the findings of CSPG production from OL cultures (Courel et al., 1998; Garwood et al., 2004; Karus et al., 2016; Niederost et al., 1999; Probstmeier et al., 2000; Szuchet et al., 2000; Yim et al., 1993) and the application of ChABC, inhibiting CSPG production or inactivation of CSPG receptor PTPRZ in purified OL cultures promote their differentiation (Karus et al., 2016; Kuboyama et al., 2015). Our mass spectrometry analyses indicate that within the OL lineage, CS-GAGs are uniquely secreted by OPCs. OL lineage progression is accompanied by a precipitous drop in CS-GAG levels. This drop appears to be an essential step in OL maturation. Our studies suggest that the drop in CS-GAGs is not likely from increased catabolism, as the $\beta$-glucuronidase activity remans comparable through maturation (Fig. S4). Rather, the expression of genes involved in CS-GAG production (identified from GO terms) decrease through OL differentiation.

We utilized LC/MS studies to analyze three GAGs (CS, HS and HA) with wellestablished roles in CNS development. We discovered that C-4S is the major GAG species secreted ( $\sim 85 \%$ of total CS-GAG) by the OL lineage (Fig. 3). Differentially sulfated CS-GAGs mediate unique biological activities (Properzi et al., 2005). The discovery of $\mathrm{C}-4 \mathrm{~S}$ production within OPCs and its auto-inhibitory activity on these cells raises important questions about CS-GAG metabolism in normal development and in the context of brain disease and repair. Despite the biological activity conferred by the GAGs in their free form and as proteoglycans, there have limited reports exploring GAG metabolism or secretion in the OL lineage. Rather, secreted proteoglycans (e.g., aggrecan family proteins) have been the major focus of studies exploring GAG/ proteoglycan significance in injury and development.

How does THAP1 LOF cause GAG accumulation? We demonstrate that GusB is the critical downstream effector for this phenotype. GusB encodes $\beta$-glucuronidase, a lysosomal enzyme that catalyzes the terminal stages of GAG catabolism by hydrolyzing glucuronide or glucuronic acid from multiple GAG species (CS-, HS- and HA-GAGs) (Heuer et al., 2001; Ray et al., 1999). Complete loss of GUSB causes Sly syndrome (MPS VII) (Beaudet et al., 1974; Sly et al., 1974), a lysosomal storage disease with marked GAG elevations (Heuer et al., 2001; Kubaski et al., 2017; Ray et al., 1999), lysosomal abnormalities (Bayo-Puxan et al., 2018; Paigen, 1989; Vogler et al., 1990) and multi-organ defects including neurodevelopmental, cognitive and motor dysfunction 
(Montano et al., 2016; Sands, 2014; Tomatsu et al., 2009). Importantly and consistent with our findings, MPS VII patients and mouse models (GusBmps) (Kumar et al., 2014) exhibit reductions in CNS myelination and altered expression of myelin component genes (Parente et al., 2012). Myelin abnormalities also occur in other MPS syndromes (Reichert et al., 2016; Seto et al., 2001). Most studies of MPS VII pathogenesis in the CNS have focused on GusB function and regulation in neurons (Bayo-Puxan et al., 2018; Levy et al., 1996; Parente et al., 2012; Parente et al., 2016; Sly and Vogler, 1997, 2002; Vogler et al., 1998). In contrast, our studies constitute the first effort defining the role of GusB in the glial lineage. Klotho, which codes for a type I membrane protein with glucuronidase activity and is related to $\beta$-glucuronidase has been shown to promote the maturation of OL lineage (Chen et al., 2013; Zeldich et al., 2015). However, Klotho is not a resident of lysosomes, has not been implicated in any MPS, and it is unknown if it plays any role GAG metabolism. Restoration of $\beta$-glucuronidase restores GAG homeostasis in the OL lineage, rescues in vitro maturation of Thap $1 \%$ OLs, and significantly improves Thap1\% CNS myelination. These observations establish lysosomal catabolism of GAGs as an essential element of THAP1 CNS function.

Most DYT6 dystonia mutations in THAP1 are predicted to cause LOF, including early stop mutations (Blanchard et al., 2011; Bragg et al., 2011). Our prior studies demonstrate that THAP1 has a prominent role in CNS myelination during juvenile developmental, the period when dystonia emerges in most DYT6 subjects (Yellajoshyula et al., 2017). These observations highlight a critical role for lysosomal GAG catabolism during CNS maturation, and implicate this pathway in the neurodevelopmental disease DYT6 dystonia. While the experiments presented here focus exclusively on GAG effects within and on the OL lineage, considerable data suggest that GAG abnormalities impact neural plasticity and function (Lin et al., 2008; Smith et al., 2015). These non-cell autonomous effects, the impact of potentially abnormal GAG metabolism within neurons themselves, and the impact of delayed OL maturation on neural function are important future directions in the study of DYT6 pathogenesis, the role of ECM abnormalities in brain injury and disease, and fundamental understanding of neuronal-glial interactions.

\section{MATERIALS \& METHODS}

\section{Generation and Maintenance of Mice}

Animal research was conducted in accord with the NIH laboratory animal care guidelines and with the Institutional Animal Care and Use Committee (IACUC) at the University of Michigan. Thap1 floxed mice generation, characterization and genotyping have been previously described (Yellajoshyula et al., 2017). Nestin-Cre+ and TgGus (Stock \# 006558) was purchased from Jackson Laboratory (Bar Harbor, ME, USA). Olig2-Cre mice were kindly provided by Dr. Roman Giger. The breeding strategy used to generate derive all primary OPC cells and conditional null mice was as follows: Thap1+-, Cre+, TgGus $\times$ Thap1floxflox. This breeding strategy produced the following offspring \pm TgGUS: Thap 1flox/+; Thap1flox/-; Thap1flox/+, Cre+; and Thap1flx/-, Cre+ (CKO). Age and sex-matched littermate mice were used for all experiments. Primers used for genotyping in this study (Thap1, Cre \& TgGUS) are listed in Table S4. 


\section{RNA extraction, qRT-PCR and RNAseq}

Total RNA extraction from OL cultures for qRT-PCR and RNAseq analysis was done using NucleoSpin® RNA (Takara) and Trizol (Thermofisher) for mouse cerebral cortex as per manufacturers instructions. cDNA synthesis from total RNA was done using MMLV Reverse Transcriptase (Takara) as per manufacturers instructions. Quantitative real time PCR (qRT-PCR) was performed with the StepOnePlus System (ABI) and 2x SYBR Power Mix (ABI). RNAseq was performed on Illumina HISeq2500 (Illumina) platform at the neurogenetics laboratory, NIA. RNAseq libraries were made using the TruSeq Stranded Total RNA Library prep kit with rRNA removal mix Gold (Illumina, 20020598). Libraries were quantified and normalized using the KAPA Library Quantification kit for Illumina Platforms (KAPA Biosystems, KR0405) and sequenced on an Illumina HiSeq 2500 using HiSeq SBS kit v4 250 cycle kit (Illumina, FC-401-4003). A standard Illumina pipeline was used to generate fastq files. RNAseq Analyses was performed using DESEQ.

\section{Immunostaining}

Immunofluorescence was done as previously described (Yellajoshyula et al., 2017) for the following antibodies: Rat anti-MBP (Millipore, Cat\# MAB386), Mouse anti-O4 (kindly provided by Dr. Roman Giger), Goat anti-OLIG2 (R\&D, AF2418), Rabbit anti-NG2 (Millipore, AB5320) and Rat anti-LAMP1 (Santacruz, SC-19992). Staining for CS and HA GAGs with Mouse anti-CS-56 (Thermofisher, MA1-83055) and Biotinylated HABP (Millipore, 385911) was performed in PBS + 1\% BSA with no detergent, unless mentioned otherwise. Quantification of staining intensity (normalized for area) was performed using imageJ analysis by tracing individual cells for CS-56, and using intensity of the entire image for HABP (to account for amorphous staining of HA-GAGs under non-permeabilizing conditions).

\section{Electron Microscopy}

EM was performed as previously described (Yellajoshyula et al., 2017). Briefly, P21 mice were anesthetized and perfused with EM perfusion solution (3\% paraformaldehyde, $2.5 \%$ glutaraldehyde in $0.1 \mathrm{M}$ phosphate buffer). Following perfusion, brains were dissected and postfixed at $4^{\circ} \mathrm{C}$ overnight in perfusion solution. Genu of CC was dissected, processed and sectioned at EM core facility, Emory University. All EM images were acquired using JEOL JSM 1400 at the University of Michigan, MIL core services. Axon caliber and g-ratio (ratio of the inner axonal diameter to total (including myelin) outer diameter) were calculated as previously described (Winters et al., 2011; Yellajoshyula et al., 2017).

\section{Derivation of OPC from NSC cells}

OPC used in this study were derived from primary NSC isolated from the sub ventricular zone (SVZ) of P7 mouse pups as previously described (Yellajoshyula et al., 2017). Routine propagation of the OPC was done as a monolayer on poly-L-ornithine $(0.1 \mathrm{mg} /$ $\mathrm{ml}$ ) and laminin $(5 \mu \mathrm{g} / \mathrm{ml})$ coated dishes in OPC expansion media (SATO medium supplemented with $20 \mu \mathrm{g} / \mathrm{ml}$ PDGF and FGF2; (Yellajoshyula et al., 2017)) or differentiated on poly-L-ornithine $(0.1 \mathrm{mg} / \mathrm{ml})$ and poly-D-lysine $(0.1 \mathrm{mg} / \mathrm{ml})$ coated 
coverslips or dishes in oligodendrocyte differentiation medium (SATO media with 40 $\mu \mathrm{g} /$ ml T3) (Yellajoshyula et al., 2017).

$\beta$-glucuronidase activity assay - MUG assay

Tissue or cell pellets were lysed in MUG Iysis buffer (0.2M Sodium acetate buffer, $\mathrm{pH}$ $4.5 ; 10 \mathrm{mM}$ EDTA; $0.1 \%$ Trixton-X-100) to obtain total cell homogenate. Equal concentrations of serially diluted lysate (in the range of $0-60 \mu \mathrm{g} / \mathrm{ml}$ ) from control and Thap1 cKO genotypes was incubated with $10 \mathrm{mM}$ 4-methylumbelliferyl $\beta$-D-glucuronide (MUG, Sigma) in $0.2 \mathrm{M}$ Sodium acetate buffer, $\mathrm{pH} 4.5$ at $37^{\circ} \mathrm{C}$ for one hour. The reaction was stopped with equivalent volume of $0.2 \mathrm{M}$ sodium citrate followed by which the fluorescence $($ excitation $=360 \mathrm{~nm}$; emission $=460 \mathrm{~nm}$ ) was measured using Biotek Synergy HT microplate reader. Based on fluorescence reading from purified $\beta$ glucuronidase standard (Sigma), we determined the $\beta$-glucuronidase activity / hour and $\beta$-glucuronidase activity / $\mathrm{mg}$ of the lysate.

\section{Quantitative Chromatin immunoprecipitation (qCHIP)}

qCHIP was performed as previously described (Yellajoshyula et al., 2011). Sheared chromatin (sonicated to 200-500 bp) from $2 \times 10^{6}$ mouse OL cells was incubated with

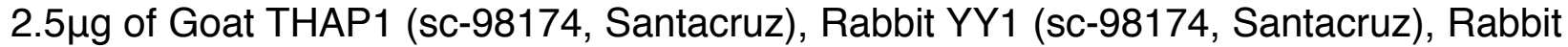
H3K4me3 (sc-98174, Activemotif), normalized Goat IgG (Santacruz), or normalized Rabbit IgG (Santacruz) using Dynabeads (Invitrogen). After washing, elution and crosslink reversal, DNA from each ChIP sample and the corresponding input sample was purified and analyzed further using qPCR. Each ChIP sample and a range of dilutions of the corresponding input sample $(0.01-2 \%$ input) were quantitatively analyzed with gene-specific primers using the StepOnePlus System $(A B I)$ and SYBR qPCR Powermix $(\mathrm{ABI})$.

\section{Gene Ontology enrichment analysis}

Candidate THAP1-dependent DEGs identified from RNAseq catalysis were analyzed for enrichment of biological pathways by gene ontology (GO) enrichment analyses using the following web based applications: GENEONTLOGY http://geneontology.org/docs/ go-enrichment-analysis/ and DAVID https://david.ncifcrf.gov/home.jsp. Top GO enriched ( $p<0.01$ and $>2$ fold enrichment) for GO-Biological processes and GO-Molecular function was used for this study.

\section{Statistics}

All data are reported as mean \pm SEM. All statistical tests reported (Student's t-tests, One-way or two-way ANOVAs) were performed using Graphpad Prism software (V9).

\section{Data \& Software Availability}

The GEO accession number for CHIP-seq data used in manuscript for THAP1 is GSM803408, YY1 is GSM803446 and H3K4me3 is GSM788087.

\section{ACKNOWLEDGEMENTS}

We thank Haoran Huang and Stephanie Mrowczynski for technical assistance. We thank Cathy Collins and Jay Li for critical reading of manuscript. We are grateful to the 
staff of UCSD Glycoanalytics Core for GRIL LC/MS analysis. We thank Dr. Herbert Geller for helpful suggestions. We thank the staff of University of Michigan's Core Facilities (DNA Sequencing Core, Microscopy and Image Analysis Laboratory and Unit of Laboratory Animal Medicine) and Hong Yi (EM Core, Emory University). This research was supported in part by the following grants: to WTD (7R56NS109227-02 NINDS).

COMPETING INTERESTS

The authors declare no competing interests. 


\section{FIGURE LEGENDS}

Figure 1. THAP1 loss disrupts the extracellular matrix transcriptome

(A-D) Loss of THAP1 causes maturation deficits in differentiating OPC. (A) Representative Images of control (Thap $1^{+/+}$) and THAP1 null (derived from Thap $1^{\mathrm{fl} / \mathrm{l} \text {-; }}$ Olig2-Cre+) OL differentiated (+T3) for 4 days (scale bar $100 \mu \mathrm{m}$ ). Cultures are stained for O4, MBP, \& DAPI. (B) Quantification of the percentage of O4+ cells expressing MBP. Bar graph shows mean values \pm SEM. Thap $1^{+/+}=86.78 \% \pm 3.98 ;$ Thap $1^{1-}=19.58 \% \pm$ 3.88; $t$-test: $t_{(4)}=12.07 ; p=0.0003 ; N=3$ clonal lines (100 cells per clone) per genotype. (C) Mean intensity of MBP staining in O4+ cells represented as arbitrary units (AU), normalized to background. Each data point in the scatter plot represents MBP+ staining intensity for individual cells. Thap $1^{+++}=10.76 \mathrm{AU} \pm 0.76$; Thap $1^{-/-}=3.32 \mathrm{AU} \pm$ 0.108 ; t-test: $\mathrm{t}_{(427)}=18.21 ; p<0.0001$. (D) Average area of $\mathrm{O} 4+$ cells. Bar graph shows mean values \pm SEM. Thap $1^{+/+}=0.043 \mathrm{~mm}^{2} \pm 0.00402$; Thap $1^{-1}=0.030 \mathrm{~mm} 2 \pm 0.00309$; t-test: $\mathrm{t}_{(633)}=2.639 ; \mathrm{p}=0.008$. (E) Schematic illustrating RNAseq analyses. Total RNA was extracted from three independent clonal lines each of Thap $1^{+/+}$and Thap $1^{-1-}$ cells at three different time points (OPC; DIV2 and DIV4) and analyzed using Illumina Hiseq (Methods). (F) Venn diagram depicting the overlap of Thap $1^{+/+}$vs Thap $1^{-/-}$differentially expressed genes (DEG) from OPC, DIV2 \& DIV4. (G) Number of differentially expressed genes (DEG) at each time point analyzed. $X$-axis $=\log _{2}$ fold change (magnitude of change for the DEG) in Thap 1 - . Y-axis $=-\log _{10}$ pAdjusted depicts the statistical significance of the change. The number of up- and down-regulated genes are indicated by arrows. (H) The DEG at either DIV2 (703) or both DIV2 \& DIV4 (277) were used for enrichment analysis of GO (Gene Ontology) terms to identify overrepresented biological pathways using both GENEONTOLOGY (http://geneontology.org) and DAVID (https://david.ncifcrf.gov/home.jsp). Graphs shows the most significantly overrepresented GO terms ( $p<0.001$, sorted as fold overrepresented) in OL from DIV2 (GENEONTOLOGY, GO - Molecular Function), and from the DIV2 \& DIV4 overlapping group (DAVID, GO - Molecular Function).

\section{Figure 2. Thap1 null oligodendrocytes accumulate and secrete excess} glycosaminoglycans

Representative images and corresponding quantification for Thap1+/+ and Thap $1^{-1-} \mathrm{OL}$ cells (DIV4) immunostained with (A, B) CS-56 (CS-GAG), and (C, D) biotinylated HABP (HA-GAG) under non-permeabilizing conditions. Thap1- OL exhibit significant accumulation of CS-GAG (Thap $1^{+/+}=6.68 \pm 1.13$; Thap $1^{-/}=12.60 \pm 1.29$; Thap $1^{-1}+$ ChABC $=3.93 \pm 0.286$; One-way ANOVA $F_{(2,205)}=14.59, p<0.0001$, Dunnett's multiple comparisons test: adjusted $p$ value $<0.0001)$ and HA-GAG (Thap1+/+ $=27.64 \mathrm{AU} \pm$ 0.38; Thap 1\%= 37.45 AU \pm 2.68 ; Thap $1 \%$ + Hyaluronidase = 26.77 AU \pm 1.16 ; One-way ANOVA $\mathrm{F}_{(2,21)}=75.59, \mathrm{p}<0.0001$, Dunnett's multiple comparisons test: adjusted $\mathrm{p}$ value $=0.0004 \&<0.0001)$. (E) Schematic illustrating the study design for mass spectrometry analyses of CS, HS and HA GAGs using GRIL LC/MS analysis. Total CS, HS and HA GAGs $(\mu \mathrm{g} / \mathrm{mg})(\mathrm{F})$ secreted in the media or (I) from cell homogenate from Thap1+/+ OPC and OL (see Fig. S2C-D for GAG content). (G-H, J-K) Abnormal accumulation of GAGs in Thap 1- cultures. Total GAGs $(\mu \mathrm{g} / \mathrm{mg})(\mathrm{CS}, \mathrm{HA}$ and HA-GAGs combined) $(G)$ secreted in the media or $(J)$ from cell homogenate from Thap $1^{+/+}$and 
Thap 1- OPC and OL (see Fig. S2C-D for GAG content). CS-GAGs $(\mu \mathrm{g} / \mathrm{mg})$ and the composition of mono (C-4S, C-6S) and bi-sulfated (C-2,6S and C-4,6S) (H) CS-GAGs secreted in the media or $(\mathrm{K})$ from cell homogenate from Thap $1^{1 /+}$ and Thap $1^{-1-}$ OPC and OL (see Fig. S2E-F for GAG content). (L-N) C-4S and C-S6S inhibit OL differentiation. Representative Images (L) and corresponding quantification $(M-N)$ of differentiated wild type OPC without (L, left column) or with C-4S $(10 \mu \mathrm{g} / \mathrm{ml}$; middle column) or C-6S $(10 \mu \mathrm{g} /$ $\mathrm{ml}$; right column) GAG applied to the culture media from DIV2 to DIV5 (scale bar 50 $\mu \mathrm{m})$. (M) Mean intensity of MBP staining at DIV5 normalized to background; Thap $1^{+/+}=$ $6.64 \mathrm{AU} \pm 0.36$; Thap1+/+ $+\mathrm{C}-4 \mathrm{~S}=2.11 \mathrm{AU} \pm 0.126$; Thap $1^{+/+}+\mathrm{C}-6 \mathrm{~S}=2.33 \mathrm{AU} \pm 0.128$; One-way ANOVA $F_{(2,681)}=119.9, p<0.0001$, Dunnett's multiple comparisons test: adjusted $p$ value $=0.0004 \&<0.0001$. Each data point in the scatter plot represents $\mathrm{MBP}+$ staining intensity for individual cells. (N) Area of O4 stained OL (Thap $1^{+/+}=8038$ $\mu \mathrm{m}^{2} \pm 463.8$; Thap $1^{+/+}+\mathrm{C}-4 \mathrm{~S}=5122 \mu \mathrm{m}^{2} \pm 230.3$; Thap $1^{+/+}+\mathrm{C}-6 \mathrm{~S}=4383 \mu \mathrm{m}^{2} \pm$ 198.6; One-way ANOVA $\mathrm{F}_{(2,627)}=34.1, \mathrm{p}<0.0001$, Dunnett's multiple comparisons test: adjusted $p$ value $=<0.0001$ ). Each data point in the scatter plot represents the area measured for individual cells.

Figure 3. Excess glycosaminoglycan secretion by Thap1 null cells impairs oligodendroglial maturation via a non-cell autonomous mechanism

(A-D) Treatment with ChABC rescues maturation defects in Thap 1- OL. (A) Representative Images (scale bar $100 \mu \mathrm{m}$ ) of Thap $1^{+/+}$and Thap $1^{1 /-}$ OL differentiated for 4 days $(+\mathrm{T} 3)$ either untreated or with ChABC from DIV2 to DIV4 $(0.1 \mathrm{U} / \mathrm{ml})$. Cultures are stained for O4, MBP, \& DAPI. (B) Quantification of the percentage of O4+ cells expressing MBP. Bar graph shows mean values \pm SEM. Thap $1^{+/+}=85.31 \% \pm 4.14$; Thap $1^{--}=20.81 \% \pm 4.76$; Thap $1^{--}+$ChABC $=56.29 \% \pm 6.31$. One-way ANOVA F $\mathrm{F}_{(2,27)}=$ 47.94, $p<0.0001$, Dunnett's multiple comparisons test: adjusted $p$ value $<0.0001$. (C) Average area of $\mathrm{O} 4+$ cells. Each data point in the scatter plot represents the area measured for individual cells. Thap $1^{+/+}=10163 \mu \mathrm{m}^{2} \pm 675.9$; Thap $1^{-/}=7667 \mu \mathrm{m}^{2} \pm$ 549.5; Thap1- + ChABC $=11401 \mu \mathrm{m}^{2} \pm 968.3$. One-way ANOVA $\mathrm{F}_{(2,309)}=6.35, \mathrm{p}=$ 0.002 , Dunnett's multiple comparisons test: adjusted $p$ value $=0.009$. (D) Mean intensity of MBP staining in O4+ cells represented as arbitrary units (AU) normalized to background. Each data point in the scatter plot represents $M B P+$ staining intensity for individual cells. Thap $1^{+/+}=11.07 \mathrm{AU} \pm 0.772$; Thap $1^{-/}=3.22 \mathrm{AU} \pm 0.124$; Thap $1^{-/}+$ $\mathrm{ChABC}=7.86 \mathrm{AU} \pm 0.63$. One-way ANOVA $\mathrm{F}_{(2,524)}=134.7, \mathrm{p}<0.0001$, Dunnett's multiple comparisons test: adjusted $p$ value $<0.0001$. (E) Schematic illustrating coculture experimental paradigm to test for paracrine effects of Thap1 null (Thap $\left.{ }^{-1}\right) \mathrm{OL}$ co-cultured with LV-GFP labelled control (Thap1+/; GFP) OPC at 10:1 ratio in differentiation media for four days (+T3). (F) Representative images (scale bar $100 \mu \mathrm{m}$ ) of Thap $1^{+/+}$;GFP OL cultured in control or mutant environments for 4 days (+T3). Cultures are stained for O4, MBP, \& DAPI. (G) MBP+ staining intensity of Thap1+/+; GFP OL. Each data point in the scatter plot represents $\mathrm{MBP}+$ staining intensity for individual cells. Thap 1+/; GFP + Control environment $=3.90 \mathrm{AU} \pm 0.146$; Thap1+/; GFP + Mutant environment $=1.286 \mathrm{AU} \pm 0.227$; Thap ${ }^{+++} ; \mathrm{GFP}+$ Mutant environment $+\mathrm{ChABC}=3.05$ $A U \pm 0.348$; One-way ANOVA $F_{(2,197)}=20.26, p<0.0001$, Dunnett's multiple comparisons test: adjusted $p$ value $<0.0001$. (H) Area of Thap1+/+; GFP OL. Each data point in the 
scatter plot represents the area measured for individual cells. Thap $1^{-/}+$Control environment $=10163 \mu \mathrm{m}^{2} \pm 675.9$; Thap $1^{--}+$Mutant environment $=7146 \mu \mathrm{m}^{2} \pm 1220$; Thap1\% + Mutant environment + ChABC $=9151 \mu \mathrm{m}^{2} \pm 1160$; One-way ANOVA $\mathrm{F}_{(2,266)}=$ 6.84, $\mathrm{p}=0.0013$, Dunnetts's multiple comparisons test: adjusted $\mathrm{p}$ value $<0.0049$.

Figure 4. THAP1 directly binds to and regulates the GusB gene encoding the GAG-catabolizing lysosomal enzyme $\beta$-glucuronidase

(A) Genome browser track (using Integrative Genomics Viewer) showing CHIP-Seq signals for THAP1, YY1 and H34me3 at the GusB locus in K652 cells (ENCODE dataset). (B-D) Quantitative ChIP (qChIP) demonstrating THAP1 dependent binding of YY1 and H3K4me3 methylation at the GusB promoter region in OL (+T3 - DIV4). Binding, represented as \% input (y-axis), is demonstrated for (B) THAP1 (x-axis) (Thap1+/+ $=0.087 \% \pm 0.008 ;$ Thap $1^{-1-}=0.02 \% \pm 0.001 ;$ t-test: $\left.\mathrm{t}_{(4)}=6.85 ; \mathrm{p}=0.02\right) ;(\mathrm{C})$ YY1 (x-axis) (Thap1+/+ $=0.23 \% \pm 0.017$; Thap1 $1-=0.018 \% \pm 0.0009$; t-test: $\mathrm{t}_{(2)}=12.85$; $\mathrm{p}=0.006$ ); and (D) H3K4me3 (x-axis) (Thap1+/+ $=2.93 \% \pm 0.219$; Thap1/- $=0.707 \% \pm$ 0.036 ; t-test: $t_{(2)}=10.04 ; p=0.0098$ ) and their respective isotype control lgG (Goat lgG or Rabbit lgG) at the GusB promoter region. Bar graph shows mean values \pm SEM. Percentage input represents the final amount of immunoprecipitated chromatin/gene from corresponding Thap1+/ and Thap1\% OL cells (Methods). (E-J) THAP1 loss significantly reduces GusB mRNA expression and $\beta$-glucuronidase enzyme activity in OLs and in CNS tissue. (E) GusB mRNA expression (qRT-PCR) from Thap1+t and Thap1- differentiating OL (OPC, DIV2 \& DIV4). GusB expression was normalized to $R$ pl19 expression and is represented as fold change (y-axis) with respect to Thap1+/. Line graphs show mean values \pm SEM. (F-G) Deficient $\beta$-glucuronidase enzyme activity in Thap1-- OPC. Line graph shows the mean value of $\beta$-glucuronidase activity ( $y$-axis = Units/Hr) for (F) Thap1+/+ and Thap1-/- OPC lysate (0 - $3 \mu \mathrm{g}$; x-axis) derived from fluorometry assay (Methods). Bar graph shows mean \pm SEM values of normalized $\beta$ glucuronidase activity Units/Lysate $(\mathrm{mg})$ for $(\mathrm{G})$ OPC (Thap1+/+ $=9.05 \mathrm{U} / \mathrm{mg} \pm 0.803$; Thap1- $=0.396 \mathrm{U} / \mathrm{mg} \pm 0.038$; t-test: $\left.\mathrm{t}_{(10)}=10.76 ; \mathrm{p}<0.0001\right)$. (H) GusB mRNA expression (qRT-PCR) from control (Thap1+/flx) and THAP1 N-CKO (Thap1flx/-; Nestin$\mathrm{Cre}^{+}$) CNS (cerebral cortex, age P21). GusB expression was normalized to Rp/19 expression and is represented as fold change (y-axis) with respect to control. Line graphs show mean values \pm SEM. (I-J) Deficient $\beta$-glucuronidase enzyme activity in THAP1 N-CKO CNS. Line graph shows the mean value of $\beta$-glucuronidase activity ( $y-$ axis = Units / Hr) for (I) control $($ Thap1+fflx $)$ and THAP1 N-CKO (Thap1flxl-; nestin-Cre $\left.{ }^{+}\right)$ cerebral cortex lysate (0 - $60 \mu \mathrm{g}$; x-axis) derived from fluorometry assay. (J) Bar graph shows mean \pm SEM values of normalized $\beta$-glucuronidase activity Units/Lysate (mg) for (J) CNS (Control = $20.43 \mathrm{U} / \mathrm{mg} \pm 2.35$; $\mathrm{N}-\mathrm{CKO}=4.90 \mathrm{U} / \mathrm{mg} \pm 0.68$; t-test: $\mathrm{t}_{(10)}=6.34$; $p<0.0001)$. 2-way ANOVA demonstrates a main effect of genotype $(P<0.01)$ for $(E, H)$ GusB mRNA expression and $(F, J) \quad \beta$-glucuronidase activity in $(E, F)$ OPC or $(H, I)$ cerebral cortex. $\left(^{*}\right)$ represents time points where significant differences exist using post hoc Sidak's multiple comparison test. (K) Representative images (scale bar $50 \mu \mathrm{m}$ ) and (L-M) corresponding quantification of Thap1+/+ and Thap1-1- OL cells (DIV4) immunostained under conditions of membrane permeabilization for CS-56 (CS-GAG; Thap1+/+ $=1.34 \pm .072 ;$ Thap1/- $=4.44 \pm 0.16$; $\mathrm{t}^{+ \text {-test: }} \mathrm{t}_{(530)}=14.51 ; \mathrm{p}<0.0001$ ) or 


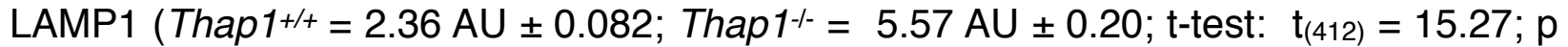
$<0.0001)$. Each data point in the scatter plot represents CS-56 or LAMP1 staining intensity for individual cells. (N) Representative images (scale bar $20 \mu \mathrm{m}$ ) of CS-GAG (CS-56) co-localization with lysosomes (LAMP1) in OL (DIV4). Pearson's coefficient value $(0.94 \pm 0.009)$ shows $\sim 95 \%$ colocalization $(R=0.94 \pm 0.009)$ (Fig. S4D).

Fig. 5. $\beta$-glucuronidase overexpression rescues the Thap1 null GAG homeostatic and OL maturation defects

(A) $\beta$-glucuronidase activity in OPC lysate from Thap1+/+, Thap1-/- and Thap1-/;TgGus genotypes (mean \pm SEM of normalized $\beta$-glucuronidase activity; Thap $1^{+/+}=7.48 \mathrm{U} / \mathrm{mg}$ \pm 0.193; Thap1-- = $0.87 \mathrm{U} / \mathrm{mg} \pm 1.29$; Thap1--; TgGUS $=304.1 \mathrm{U} / \mathrm{mg} \pm 1.6$; One-way ANOVA $F_{(2,149972)}=34628.96, p<0.0001$, Dunnett's multiple comparisons test: adjusted $p$ value $<0.0001$ ). (B) Representative images (scale bar $50 \mu \mathrm{m}$ ) of Thap1 ${ }^{-/} ; \mathrm{Tg}^{\mathrm{GUS}}$ OPC expressing NG2 (CSPG4) and pan-OL lineage marker OLIG2. (C-D) $\beta$-glucuronidase overexpression prevents CS-GAG accumulation in Thap1\% OL. (C) Representative images (scale bar $50 \mu \mathrm{m}$ ) and (D) corresponding quantification for Thap1+/, Thap1-- and Thap1--;TgGuS OL (+T3 - DIV5) immunostained with CS-56 (CS-GAG) under nonpermeabilized conditions. Each data point in the scatter plot represents CS-56 staining intensity for individual cells normalized to area. Thap $1^{+/+}=2.46 \mathrm{AU} \pm 0.35$; Thap $^{-1-}=$ 5.7 AU \pm 0.67; Thap1--;TgGUS $=1.909$ AU \pm 0.13 ; One-way ANOVA $F_{(2,34)}=19.95$ $p<0.0001$, Dunnett's multiple comparisons test: adjusted $p$ value $<0.0001$. (E-F) $\beta$ glucuronidase overexpression rescues maturation defects in Thap 1 - OL. (E)

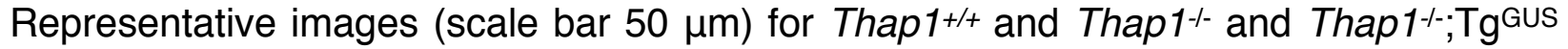
OL differentiated for 5 days (+T3). Cultures stained with O4, MBP, \& DAPI. (F) Quantification of the \% of $\mathrm{O} 4+$ cells expressing MBP and intensity of MBP staining. (F, upper graph) Percentage of $\mathrm{O} 4+$ cells expressing MBP. Bar graph shows mean \pm SEM values. y-axis; Thap $1^{++}=83.48 \% \pm 5.08$; Thap $1^{--}=26.17 \% \pm 5.33$; Thap $1^{-/}$; TgGUS $=$ $65.18 \pm 4.09$; One-way ANOVA $F_{(2,49)}=23.27, p<0.0001$, Dunnett's multiple comparisons test: adjusted $p$ value $<0.0001$. ( $F$, lower graph) Mean intensity of MBP staining normalized to background. Each data point in the scatter plot represents $\mathrm{MBP}+$ staining intensity for individual cells. Thap $1^{+/+}=4.81 \mathrm{AU} \pm 0.24$; Thap1/- $=1.005 \pm 0.004$; Thap1- $;$ TgGUS $=1.933 \pm 0.128$; One-way ANOVA $F_{(2,1195)}=127.8, p<0.0001$, Dunnett's multiple comparisons test: adjusted $p$ value $<0.0001$.

Fig. 6. $\beta$-glucuronidase overexpression rescues the myelination defect in the THAP1 null CNS.

(A) Representative transmission EM images (scale bar $4 \mu \mathrm{m}$ ) of the genu of corpus callosum at P21 from control (Thap1+/+; nestin-Cre-), control-TgGUS (Thap1+/+; nestin-

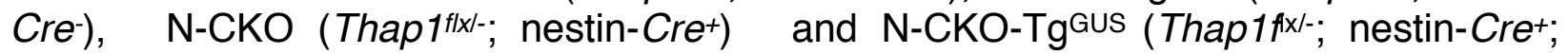
TgGUS). (B-E) Quantification of EM images ( $N=3$ per genotype) demonstrates that $\beta$ glucuronidase overexpression (N-CKO; TgGUS) significantly increases myelination in the THAP1 null CNS. (B) Density of myelinated axons (number of axons / $\mathrm{mm}^{2}$ ). Mean \pm SEM; Control = $238.09 \pm 12.58$, Control; TgGUS $=289.85 \pm 10.36 ; \mathrm{N}-\mathrm{CKO}=51.74 \pm 2.70$; $\mathrm{N}-\mathrm{CKO}$;TgGus $100.71 \pm 4.790 ;$ One-way ANOVA $\mathrm{F}_{(3,6)}=182.7, \mathrm{p}<0.0001$, Dunnett's multiple comparisons test: adjusted $p$ value $<0.0001$. (C-D) g-ratio represented in relation to axon caliber $(C)$ and by genotype $(D$, mean $\pm S E M$; Control $=0.684 \pm 0.009$, 
Control;TgGUS $=0.690 \pm 0.0087 ; \mathrm{N}-\mathrm{CKO}=0.755 \pm 0.0082 ; \mathrm{N}-\mathrm{CKO} ; \mathrm{TgGUS}=0.666 \pm$ 0.0119; One-way ANOVA $F_{(3,8)}=15.68, p=0.001$, Dunnett's multiple comparisons test: adjusted $p$ value $<0.004$ ). (E) Axon caliber (each data point represents axon caliber $(\mathrm{nm})$ of individual axons; Control $=813.2 \mathrm{~nm} \pm 14.76$, Control; $\mathrm{Tg}$ GUS $=825.9 \mathrm{~nm} \pm$ $19.54 ; \mathrm{N}-\mathrm{CKO}=935.0 \pm 21.42 ; \mathrm{N}-\mathrm{CKO} ; \mathrm{Tg}^{\mathrm{GUS}}=897.4 \pm 21.08$; One-way ANOVA $\mathrm{F}_{(3,1047)}=8.613, \mathrm{p}=0.001$, Dunnett's multiple comparisons test: adjusted $\mathrm{p}$ value < 0.004). (F) Representative EM images (scale bar $200 \mathrm{~nm}$ ) demonstrating rescue of myelin thickness (g-ratio) observed in $\mathrm{N}$-CKO with $\beta$-glucuronidase overexpression $(\mathrm{N}$ CKO; TgGUS). 


\section{REFERENCES}

Almeida, R.G., and Lyons, D.A. (2017). On Myelinated Axon Plasticity and Neuronal Circuit Formation and Function. J Neurosci 37, 10023-10034.

Asher, R.A., Morgenstern, D.A., Fidler, P.S., Adcock, K.H., Oohira, A., Braistead, J.E., Levine, J.M., Margolis, R.U., Rogers, J.H., and Fawcett, J.W. (2000). Neurocan is upregulated in injured brain and in cytokine-treated astrocytes. J Neurosci 20 , 2427-2438.

Bacmeister, C.M., Barr, H.J., McClain, C.R., Thornton, M.A., Nettles, D., Welle, C.G., and Hughes, E.G. (2020). Motor learning promotes remyelination via new and surviving oligodendrocytes. Nat Neurosci 23, 819-831.

Bayo-Puxan, N., Terrasso, A.P., Creyssels, S., Simao, D., Begon-Pescia, C., Lavigne, M., Salinas, S., Bernex, F., Bosch, A., Kalatzis, V., et al. (2018). Lysosomal and network alterations in human mucopolysaccharidosis type VII iPSC-derived neurons. Sci Rep 8, 16644.

Beaudet, A.L., Ferrante, N.M., Ferry, G.D., and Nichols, B.L., Jr. (1974). Betaglucuronidase deficiency (mucopolysaccharidosis type VII). Birth Defects Orig Artic Ser 10, 246-250.

Berezin, V., Walmod, P.S., Filippov, M., and Dityatev, A. (2014). Targeting of ECM molecules and their metabolizing enzymes and receptors for the treatment of CNS diseases. Prog Brain Res 214, 353-388.

Bergles, D.E., and Richardson, W.D. (2015). Oligodendrocyte Development and Plasticity. Cold Spring Harb Perspect Biol 8, a020453.

Blanchard, A., Ea, V., Roubertie, A., Martin, M., Coquart, C., Claustres, M., Beroud, C., and Collod-Beroud, G. (2011). DYT6 dystonia: review of the literature and creation of the UMD Locus-Specific Database (LSDB) for mutations in the THAP1 gene. Hum Mutat 32, 1213-1224.

Bragg, D.C., Armata, I.A., Nery, F.C., Breakefield, X.O., and Sharma, N. (2011). Molecular pathways in dystonia. Neurobiol Dis 42, 136-147.

Chen, C.D., Sloane, J.A., Li, H., Aytan, N., Giannaris, E.L., Zeldich, E., Hinman, J.D., Dedeoglu, A., Rosene, D.L., Bansal, R., et al. (2013). The antiaging protein Klotho enhances oligodendrocyte maturation and myelination of the CNS. J Neurosci 33, 1927-1939.

Colognato, H., and Tzvetanova, I.D. (2011). Glia unglued: how signals from the extracellular matrix regulate the development of myelinating glia. Dev Neurobiol 71, 924-955.

Courel, M.N., Marret, S., Girard, N., Chauzy, C., Olivier, A., Bertrand, P., Delpech, A., Laquerriere, A., Asou, H., and Delpech, B. (1998). Hyaluronectin is produced by oligodendrocytes and Schwann cells in vitro. J Neurocytol 27, 27-32.

Elbaz, B., and Popko, B. (2019). Molecular Control of Oligodendrocyte Development. Trends Neurosci 42, 263-277.

Esko, J.D., Kimata, K., and Lindahl, U. (2009). Proteoglycans and Sulfated Glycosaminoglycans. In Essentials of Glycobiology, nd, A. Varki, R.D. Cummings, J.D. Esko, H.H. Freeze, P. Stanley, C.R. Bertozzi, G.W. Hart, and M.E. Etzler, eds. (Cold Spring Harbor (NY)). 
Fawcett, J.W., Oohashi, T., and Pizzorusso, T. (2019). The roles of perineuronal nets and the perinodal extracellular matrix in neuronal function. Nat Rev Neurosci 20, 451-465.

Feliu, A., Mestre, L., Carrillo-Salinas, F.J., Yong, V.W., Mecha, M., and Guaza, C. (2020). 2-arachidonoylglycerol reduces chondroitin sulphate proteoglycan production by astrocytes and enhances oligodendrocyte differentiation under inhibitory conditions. Glia 68, 1255-1273.

Fields, R.D. (2008). White matter in learning, cognition and psychiatric disorders. Trends Neurosci 31, 361-370.

Fiorenza, M.T., Moro, E., and Erickson, R.P. (2018). The pathogenesis of lysosomal storage disorders: beyond the engorgement of lysosomes to abnormal development and neuroinflammation. Hum Mol Genet.

Fuchs, T., Gavarini, S., Saunders-Pullman, R., Raymond, D., Ehrlich, M.E., Bressman, S.B., and Ozelius, L.J. (2009). Mutations in the THAP1 gene are responsible for DYT6 primary torsion dystonia. Nature genetics 41, 286-288.

Garwood, J., Garcion, E., Dobbertin, A., Heck, N., Calco, V., ffrench-Constant, C., and Faissner, A. (2004). The extracellular matrix glycoprotein Tenascin- $C$ is expressed by oligodendrocyte precursor cells and required for the regulation of maturation rate, survival and responsiveness to platelet-derived growth factor. Eur J Neurosci 20, 2524-2540.

Harlow, D.E., and Macklin, W.B. (2014). Inhibitors of myelination: ECM changes, CSPGs and PTPs. Exp Neurol 251, 39-46.

Heuer, G.G., Skorupa, A.F., Prasad Alur, R.K., Jiang, K., and Wolfe, J.H. (2001). Accumulation of abnormal amounts of glycosaminoglycans in murine mucopolysaccharidosis type VII neural progenitor cells does not alter the growth rate or efficiency of differentiation into neurons. Mol Cell Neurosci 17, 167-178.

Jones, L.L., Margolis, R.U., and Tuszynski, M.H. (2003). The chondroitin sulfate proteoglycans neurocan, brevican, phosphacan, and versican are differentially regulated following spinal cord injury. Exp Neurol 182, 399-411.

Karus, M., Ulc, A., Ehrlich, M., Czopka, T., Hennen, E., Fischer, J., Mizhorova, M., Qamar, N., Brustle, O., and Faissner, A. (2016). Regulation of oligodendrocyte precursor maintenance by chondroitin sulphate glycosaminoglycans. Glia 64, 270-286. Keough, M.B., Rogers, J.A., Zhang, P., Jensen, S.K., Stephenson, E.L., Chen, T., Hurlbert, M.G., Lau, L.W., Rawji, K.S., Plemel, J.R., et al. (2016). An inhibitor of chondroitin sulfate proteoglycan synthesis promotes central nervous system remyelination. Nat Commun 7, 11312.

Kobayashi, H. (2019). Recent trends in mucopolysaccharidosis research. J Hum Genet 64, 127-137.

Kubaski, F., Brusius-Facchin, A.C., Mason, R.W., Patel, P., Burin, M.G., Michelin-Tirelli, K., Kessler, R.G., Bender, F., Leistner-Segal, S., Moreno, C.A., et al. (2017). Elevation of glycosaminoglycans in the amniotic fluid of a fetus with mucopolysaccharidosis VII. Prenat Diagn 37, 435-439.

Kuboyama, K., Fujikawa, A., Suzuki, R., and Noda, M. (2015). Inactivation of Protein Tyrosine Phosphatase Receptor Type Z by Pleiotrophin Promotes Remyelination through Activation of Differentiation of Oligodendrocyte Precursor Cells. J Neurosci 35, 12162-12171. 
Kuboyama, K., Fujikawa, A., Suzuki, R., Tanga, N., and Noda, M. (2016). Role of Chondroitin Sulfate (CS) Modification in the Regulation of Protein-tyrosine Phosphatase Receptor Type Z (PTPRZ) Activity: PLEIOTROPHIN-PTPRZ-A SIGNALING IS INVOLVED IN OLIGODENDROCYTE DIFFERENTIATION. J Biol Chem 291, 18117-18128.

Kuboyama, K., Tanga, N., Suzuki, R., Fujikawa, A., and Noda, M. (2017). Protamine neutralizes chondroitin sulfate proteoglycan-mediated inhibition of oligodendrocyte differentiation. PLoS One 12, e0189164.

Kumar, M., Nasrallah, I.M., Kim, S., Ittyerah, R., Pickup, S., Li, J., Parente, M.K., Wolfe, J.H., and Poptani, H. (2014). High-resolution magnetic resonance microscopy and diffusion tensor imaging to assess brain structural abnormalities in the murine mucopolysaccharidosis VII model. J Neuropathol Exp Neurol 73, 39-49.

Kyle, J.W., Birkenmeier, E.H., Gwynn, B., Vogler, C., Hoppe, P.C., Hoffmann, J.W., and Sly, W.S. (1990). Correction of murine mucopolysaccharidosis VII by a human betaglucuronidase transgene. Proc Natl Acad Sci U S A 87, 3914-3918.

Laabs, T.L., Wang, H., Katagiri, Y., McCann, T., Fawcett, J.W., and Geller, H.M. (2007). Inhibiting glycosaminoglycan chain polymerization decreases the inhibitory activity of astrocyte-derived chondroitin sulfate proteoglycans. J Neurosci 27, 14494-14501.

Lau, L.W., Keough, M.B., Haylock-Jacobs, S., Cua, R., Doring, A., Sloka, S., Stirling, D.P., Rivest, S., and Yong, V.W. (2012). Chondroitin sulfate proteoglycans in demyelinated lesions impair remyelination. Ann Neurol 72, 419-432.

Lawrence, R., Olson, S.K., Steele, R.E., Wang, L., Warrior, R., Cummings, R.D., and Esko, J.D. (2008). Evolutionary differences in glycosaminoglycan fine structure detected by quantitative glycan reductive isotope labeling. J Biol Chem 283, 33674-33684.

Levy, B., Galvin, N., Vogler, C., Birkenmeier, E.H., and Sly, W.S. (1996). Neuropathology of murine mucopolysaccharidosis type VII. Acta Neuropathol 92, 562-568.

Lin, R., Kwok, J.C., Crespo, D., and Fawcett, J.W. (2008). Chondroitinase ABC has a long-lasting effect on chondroitin sulphate glycosaminoglycan content in the injured rat brain. J Neurochem 104, 400-408.

McKenzie, I.A., Ohayon, D., Li, H., de Faria, J.P., Emery, B., Tohyama, K., and Richardson, W.D. (2014). Motor skill learning requires active central myelination.

Science 346, 318-322.

McKeon, R.J., Jurynec, M.J., and Buck, C.R. (1999). The chondroitin sulfate proteoglycans neurocan and phosphacan are expressed by reactive astrocytes in the chronic CNS glial scar. J Neurosci 19, 10778-10788.

Montano, A.M., Lock-Hock, N., Steiner, R.D., Graham, B.H., Szlago, M., Greenstein, R., Pineda, M., Gonzalez-Meneses, A., Coker, M., Bartholomew, D., et al. (2016). Clinical course of sly syndrome (mucopolysaccharidosis type VII). J Med Genet 53, 403-418. Niederost, B.P., Zimmermann, D.R., Schwab, M.E., and Bandtlow, C.E. (1999). Bovine CNS myelin contains neurite growth-inhibitory activity associated with chondroitin sulfate proteoglycans. J Neurosci 19, 8979-8989.

Nowicka, D., and Greda, A. (2019). Chondroitin sulfate metabolism in the brain. Acta Neurobiol Exp (Wars) 79, 338-351.

Paigen, K. (1989). Mammalian beta-glucuronidase: genetics, molecular biology, and cell biology. Prog Nucleic Acid Res Mol Biol 37, 155-205. 
Parente, M.K., Rozen, R., Cearley, C.N., and Wolfe, J.H. (2012). Dysregulation of gene expression in a lysosomal storage disease varies between brain regions implicating unexpected mechanisms of neuropathology. PLoS One 7, e32419.

Parente, M.K., Rozen, R., Seeholzer, S.H., and Wolfe, J.H. (2016). Integrated analysis of proteome and transcriptome changes in the mucopolysaccharidosis type VII mouse hippocampus. Mol Genet Metab 118, 41-54.

Pendleton, J.C., Shamblott, M.J., Gary, D.S., Belegu, V., Hurtado, A., Malone, M.L., and McDonald, J.W. (2013). Chondroitin sulfate proteoglycans inhibit oligodendrocyte myelination through PTPsigma. Exp Neurol 247, 113-121.

Pepper, R.E., Pitman, K.A., Cullen, C.L., and Young, K.M. (2018). How Do Cells of the Oligodendrocyte Lineage Affect Neuronal Circuits to Influence Motor Function, Memory and Mood? Front Cell Neurosci 12, 399.

Probstmeier, R., Stichel, C.C., Muller, H.W., Asou, H., and Pesheva, P. (2000).

Chondroitin sulfates expressed on oligodendrocyte-derived tenascin- $R$ are involved in neural cell recognition. Functional implications during CNS development and regeneration. J Neurosci Res 60, 21-36.

Properzi, F., Carulli, D., Asher, R.A., Muir, E., Camargo, L.M., van Kuppevelt, T.H., ten Dam, G.B., Furukawa, Y., Mikami, T., Sugahara, K., et al. (2005). Chondroitin 6-sulphate synthesis is up-regulated in injured CNS, induced by injury-related cytokines and enhanced in axon-growth inhibitory glia. Eur J Neurosci 21, 378-390. Properzi, F., Lin, R., Kwok, J., Naidu, M., van Kuppevelt, T.H., Ten Dam, G.B., Camargo, L.M., Raha-Chowdhury, R., Furukawa, Y., Mikami, T., et al. (2008). Heparan sulphate proteoglycans in glia and in the normal and injured CNS: expression of sulphotransferases and changes in sulphation. Eur J Neurosci 27, 593-604.

Pu, A., Stephenson, E.L., and Yong, V.W. (2018). The extracellular matrix: Focus on oligodendrocyte biology and targeting CSPGs for remyelination therapies. Glia. Rainero, E. (2016). Extracellular matrix endocytosis in controlling matrix turnover and beyond: emerging roles in cancer. Biochem Soc Trans 44, 1347-1354.

Rappaport, J., Manthe, R.L., Solomon, M., Garnacho, C., and Muro, S. (2016). A Comparative Study on the Alterations of Endocytic Pathways in Multiple Lysosomal Storage Disorders. Mol Pharm 13, 357-368.

Ray, J., Scarpino, V., Laing, C., and Haskins, M.E. (1999). Biochemical basis of the beta-glucuronidase gene defect causing canine mucopolysaccharidosis VII. J Hered 90 , 119-123.

Reichert, R., Campos, L.G., Vairo, F., de Souza, C.F., Perez, J.A., Duarte, J.A., Leiria, F.A., Anes, M., and Vedolin, L.M. (2016). Neuroimaging Findings in Patients with Mucopolysaccharidosis: What You Really Need to Know. Radiographics 36, 1448-1462. Rice, D., and Barone, S., Jr. (2000). Critical periods of vulnerability for the developing nervous system: evidence from humans and animal models. Environ Health Perspect 108 Suppl 3, 511-533.

Sands, M.S. (2014). Mucopolysaccharidosis type VII: A powerful experimental system and therapeutic challenge. Pediatr Endocrinol Rev 12 Suppl 1, 159-165.

Sandvig, A., Berry, M., Barrett, L.B., Butt, A., and Logan, A. (2004). Myelin-, reactive glia-, and scar-derived CNS axon growth inhibitors: expression, receptor signaling, and correlation with axon regeneration. Glia 46, 225-251. 
Seto, T., Kono, K., Morimoto, K., Inoue, Y., Shintaku, H., Hattori, H., Matsuoka, O., Yamano, T., and Tanaka, A. (2001). Brain magnetic resonance imaging in 23 patients with mucopolysaccharidoses and the effect of bone marrow transplantation. Ann Neurol 50, 79-92.

Siebert, J.R., and Osterhout, D.J. (2011). The inhibitory effects of chondroitin sulfate proteoglycans on oligodendrocytes. J Neurochem 119, 176-188.

Sloane, J.A., Batt, C., Ma, Y., Harris, Z.M., Trapp, B., and Vartanian, T. (2010). Hyaluronan blocks oligodendrocyte progenitor maturation and remyelination through TLR2. Proc Natl Acad Sci U S A 107, 11555-11560.

Sly, W.S., Brot, F.E., Glaser, J., Stahl, P.D., Quinton, B.A., Rimoin, D.L., and McAlister, W.H. (1974). Beta-glucuronidase deficiency mucopolysaccharidosis. Birth Defects Orig Artic Ser 10, 239-245.

Sly, W.S., and Vogler, C. (1997). Gene therapy for lysosomal storage disease: a nobrainer? Transplants of fibroblasts secreting high levels of beta-glucuronidase decrease lesions in the brains of mice with Sly syndrome, a lysosomal storage disease. Nat Med 3, 719-720.

Sly, W.S., and Vogler, C. (2002). Brain-directed gene therapy for lysosomal storage disease: going well beyond the blood- brain barrier. Proc Natl Acad Sci U S A 99, 5760-5762.

Smith, P.D., Coulson-Thomas, V.J., Foscarin, S., Kwok, J.C., and Fawcett, J.W. (2015). "GAG-ing with the neuron": The role of glycosaminoglycan patterning in the central nervous system. Exp Neurol 274, 100-114.

Song, I., and Dityatev, A. (2018). Crosstalk between glia, extracellular matrix and neurons. Brain Res Bull 136, 101-108.

Szuchet, S., Watanabe, K., and Yamaguchi, Y. (2000). Differentiation/regeneration of oligodendrocytes entails the assembly of a cell-associated matrix. Int J Dev Neurosci 18, 705-720.

Tang, X., Davies, J.E., and Davies, S.J. (2003). Changes in distribution, cell associations, and protein expression levels of NG2, neurocan, phosphacan, brevican, versican V2, and tenascin- $C$ during acute to chronic maturation of spinal cord scar tissue. J Neurosci Res 71, 427-444.

Theocharidis, U., Long, K., ffrench-Constant, C., and Faissner, A. (2014). Regulation of the neural stem cell compartment by extracellular matrix constituents. Prog Brain Res 214, 3-28.

Tomatsu, S., Montano, A.M., Dung, V.C., Grubb, J.H., and Sly, W.S. (2009). Mutations and polymorphisms in GUSB gene in mucopolysaccharidosis VII (Sly Syndrome). Hum Mutat 30, 511-519.

Vogler, C., Birkenmeier, E.H., Sly, W.S., Levy, B., Pegors, C., Kyle, J.W., and Beamer, W.G. (1990). A murine model of mucopolysaccharidosis VII. Gross and microscopic findings in beta-glucuronidase-deficient mice. Am J Pathol 136, 207-217. Vogler, C., Sands, M.S., Galvin, N., Levy, B., Thorpe, C., Barker, J., and Sly, W.S. (1998). Murine mucopolysaccharidosis type VII: the impact of therapies on the clinical course and pathology in a murine model of lysosomal storage disease. $\mathrm{J}$ Inherit Metab Dis $21,575-586$. 
Wang, F., Yang, Y.J., Yang, N., Chen, X.J., Huang, N.X., Zhang, J., Wu, Y., Liu, Z., Gao, X., Li, T., et al. (2018). Enhancing Oligodendrocyte Myelination Rescues Synaptic Loss and Improves Functional Recovery after Chronic Hypoxia. Neuron 99, 689-701 e685. Waxman, S.G., and Bennett, M.V. (1972). Relative conduction velocities of small myelinated and non-myelinated fibres in the central nervous system. Nat New Biol 238, 217-219.

Winchester, B. (2005). Lysosomal metabolism of glycoproteins. Glycobiology 15, 1R-15R.

Winters, J.J., Ferguson, C.J., Lenk, G.M., Giger-Mateeva, V.I., Shrager, P., Meisler, M.H., and Giger, R.J. (2011). Congenital CNS hypomyelination in the Fig4 null mouse is rescued by neuronal expression of the $\mathrm{PI}(3,5) \mathrm{P}(2)$ phosphatase Fig4. J Neurosci 31 , 17736-17751.

Xiao, L., Ohayon, D., McKenzie, I.A., Sinclair-Wilson, A., Wright, J.L., Fudge, A.D., Emery, B., Li, H., and Richardson, W.D. (2016). Rapid production of new oligodendrocytes is required in the earliest stages of motor-skill learning. Nat Neurosci 19, 1210-1217.

Yellajoshyula, D., Liang, C.C., Pappas, S.S., Penati, S., Yang, A., Mecano, R., Kumaran, R., Jou, S., Cookson, M.R., and Dauer, W.T. (2017). The DYT6 Dystonia Protein THAP1 Regulates Myelination within the Oligodendrocyte Lineage. Dev Cell 42, 52-67 e54.

Yellajoshyula, D., Patterson, E.S., Elitt, M.S., and Kroll, K.L. (2011). Geminin promotes neural fate acquisition of embryonic stem cells by maintaining chromatin in an accessible and hyperacetylated state. Proc Natl Acad Sci U S A 108, 3294-3299. Yim, S.H., Sherin, J.E., and Szuchet, S. (1993). Oligodendrocyte proteoglycans: modulation by cell-substratum adhesion. J Neurosci Res 34, 401-413. Zeldich, E., Chen, C.D., Avila, R., Medicetty, S., and Abraham, C.R. (2015). The AntiAging Protein Klotho Enhances Remyelination Following Cuprizone-Induced Demyelination. J Mol Neurosci 57, 185-196.

Zielonka, M., Garbade, S.F., Kolker, S., Hoffmann, G.F., and Ries, M. (2017). Quantitative clinical characteristics of 53 patients with MPS VII: a cross-sectional analysis. Genet Med 19, 983-988. 
A
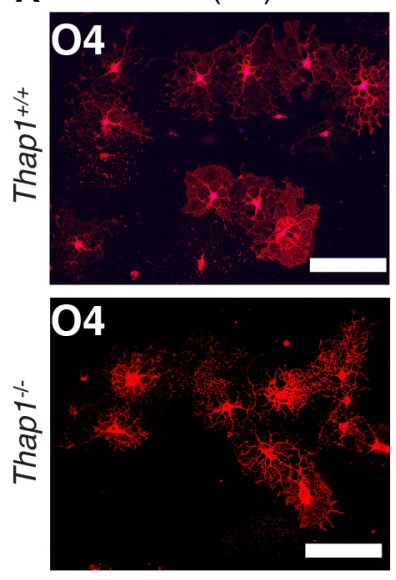

MBP (Mature OL)
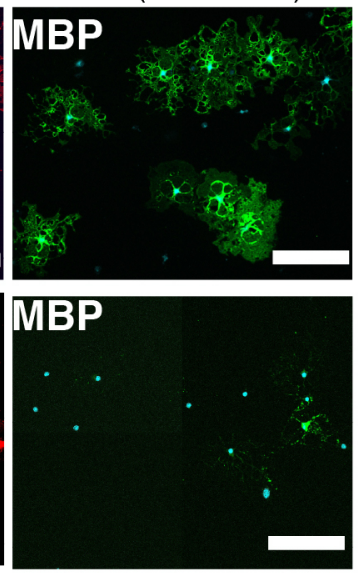

$\mathbf{F}$

THAP1 regulated genes

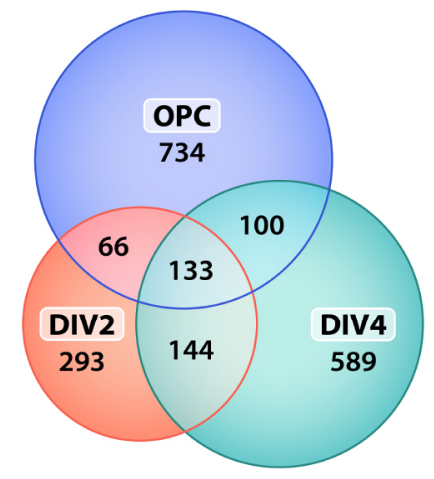

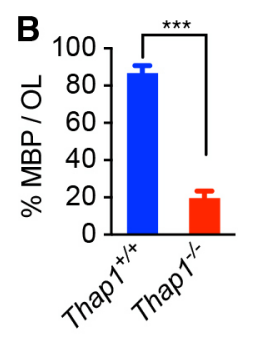
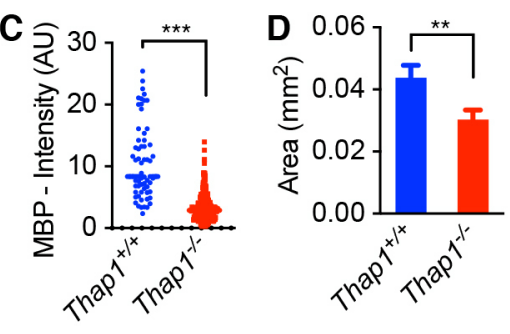

E

$$
\text { OPC }
$$

DIV2

DIV4

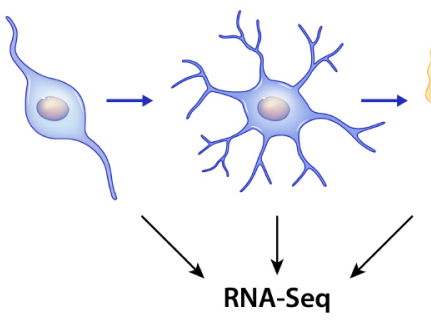

G Differentially Expressed Genes (DEG)

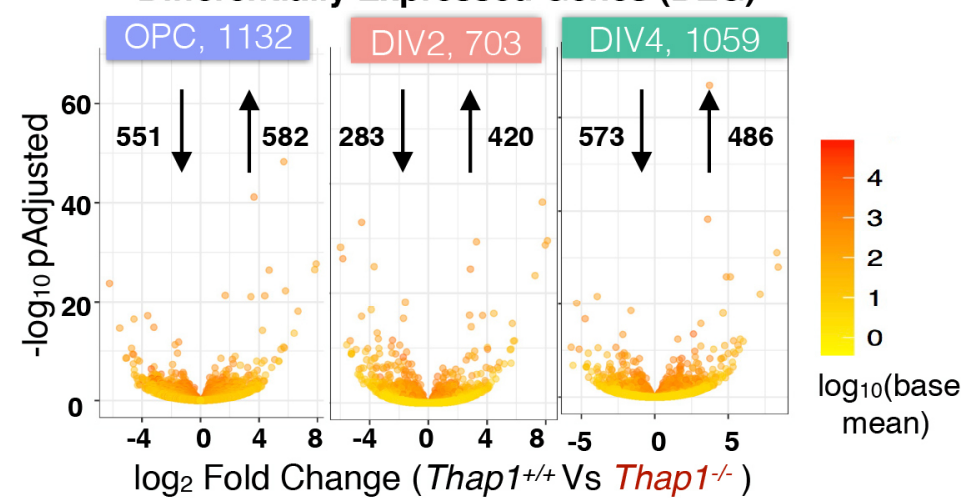

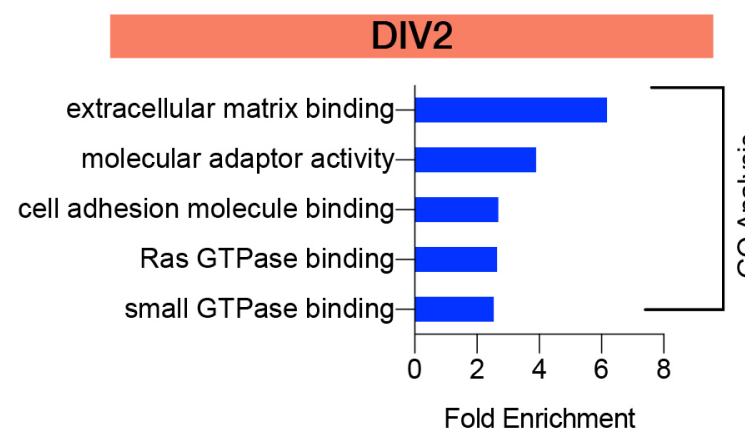

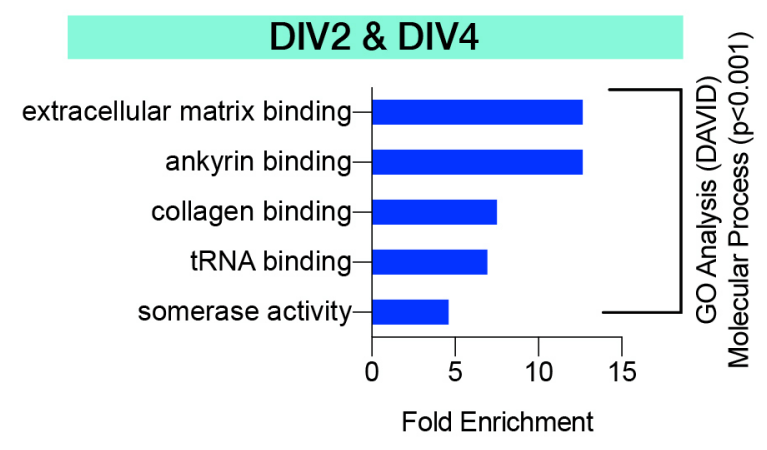


A

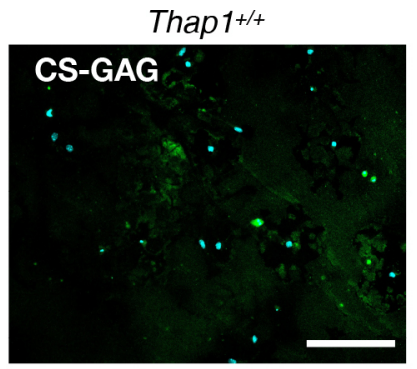

C

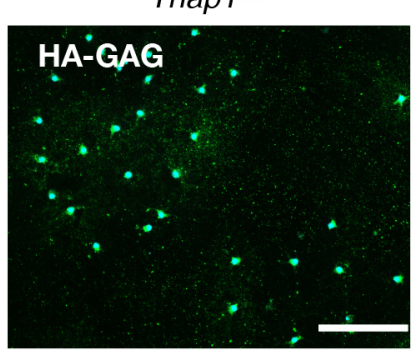

Thap 1-

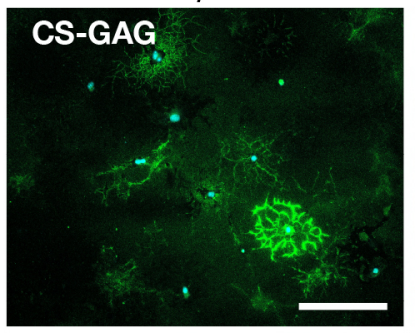

Thap 1 -

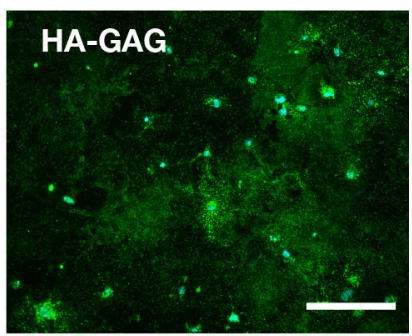

Thap 1- + ChABC

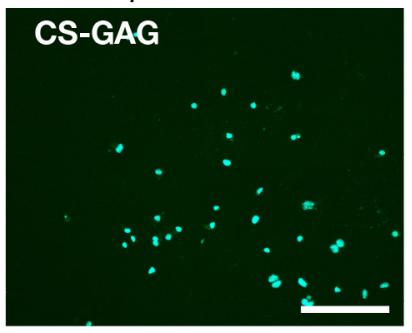

Thap $1 \%+$ Hyaluronidase

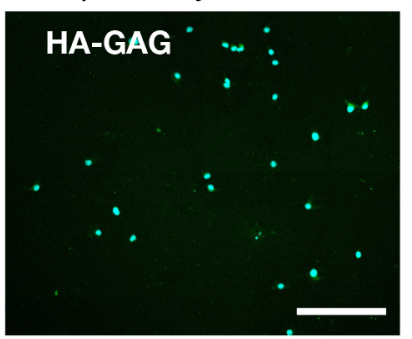

B

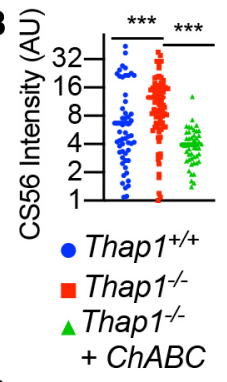

D

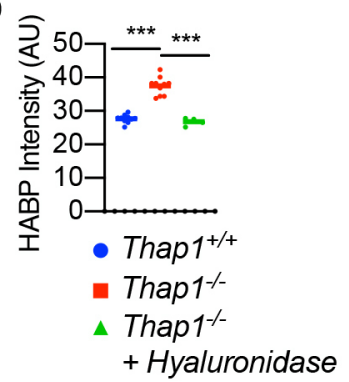

E

GAG analysis from OL culture
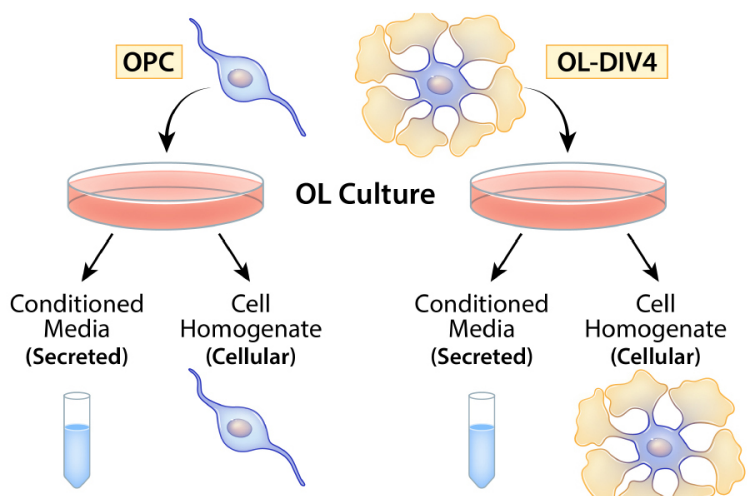

+Chase $A B C$

HPnase I-III Hyaluronidase

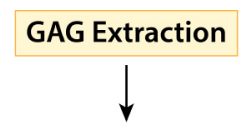

Aniline Tagging

Analysis on LC/MS
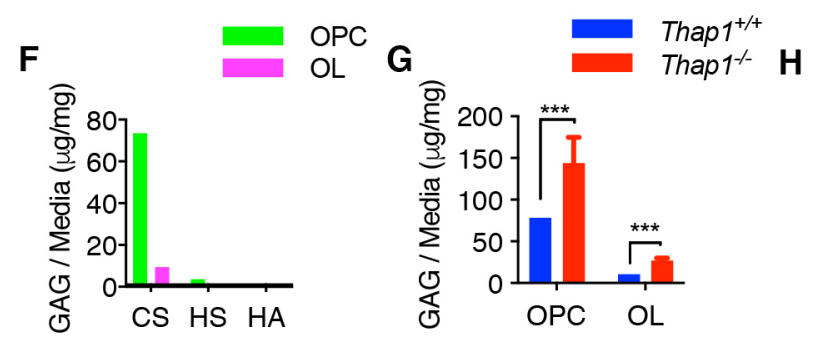

Cellular GAG (cell homogenate)

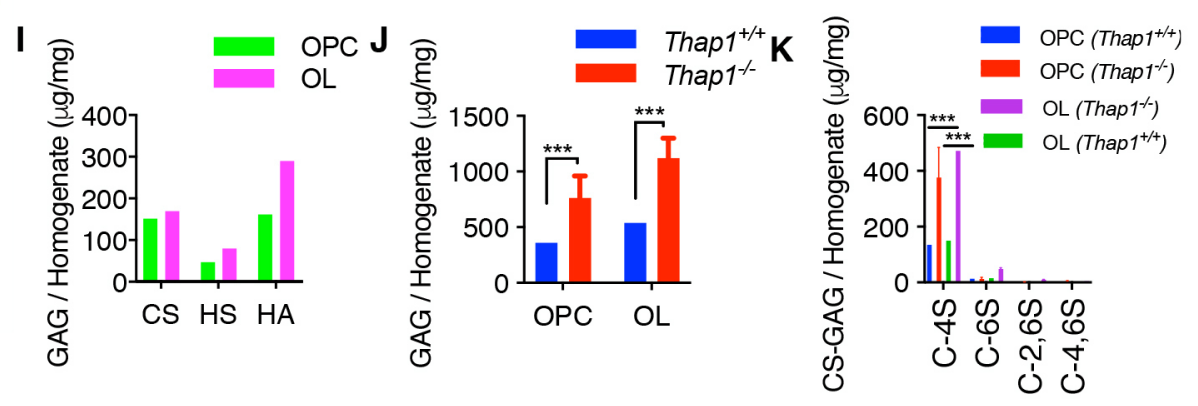

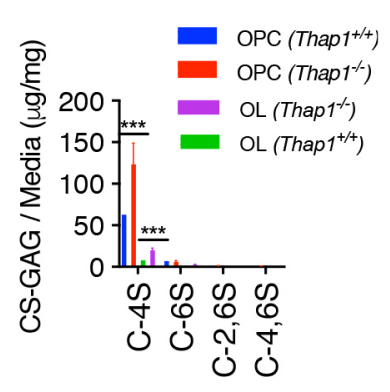

\section{Secreted GAG (media)}

\section{L}
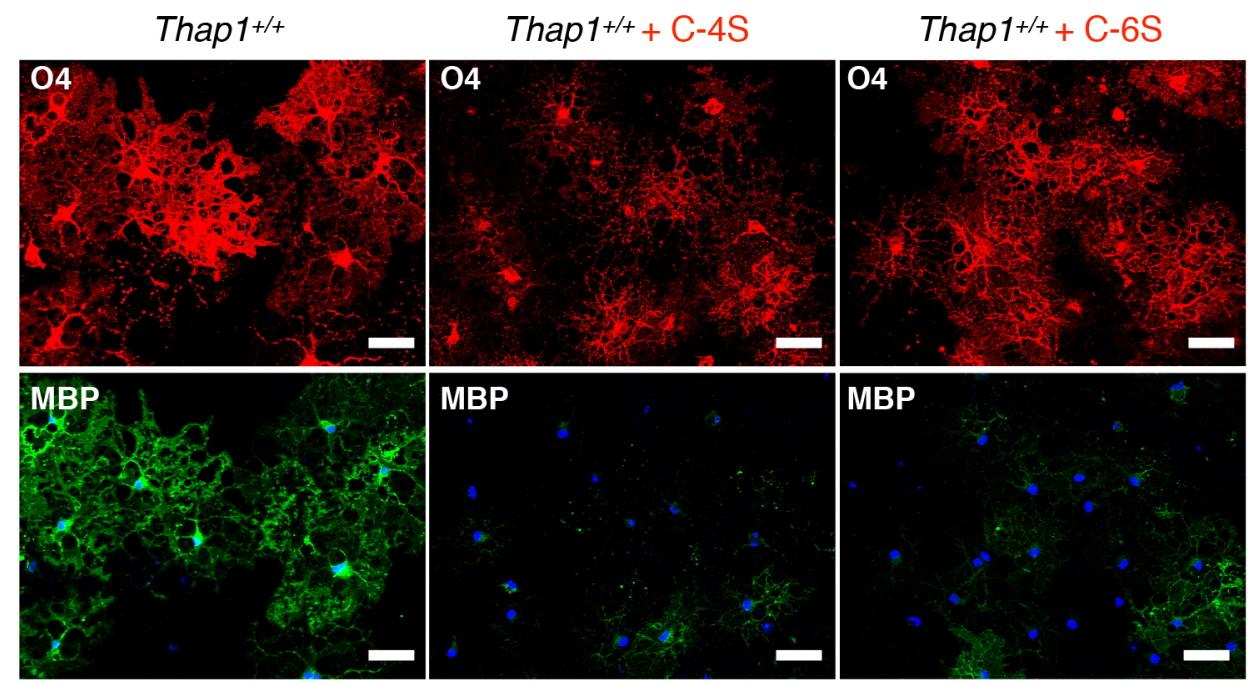

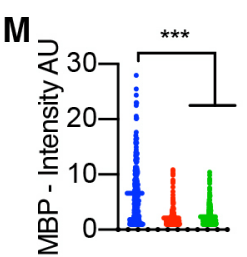

N

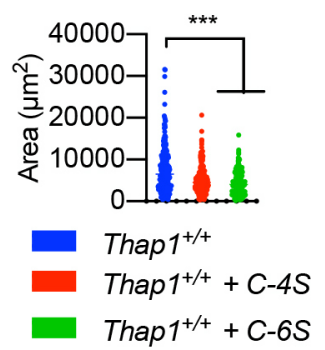


bioRxiv preprint doi: https://doi.org/10.1101/2020.09.27.316133; this version posted October 20, 2020. The copyright holder for this preprint (which was not certified by peer review) is the author/funder. All rights reserved. No reuse allowed without permission.

Figure. 3

A Thap1+/+ Thap1Thap $1 \%+$ ChABC
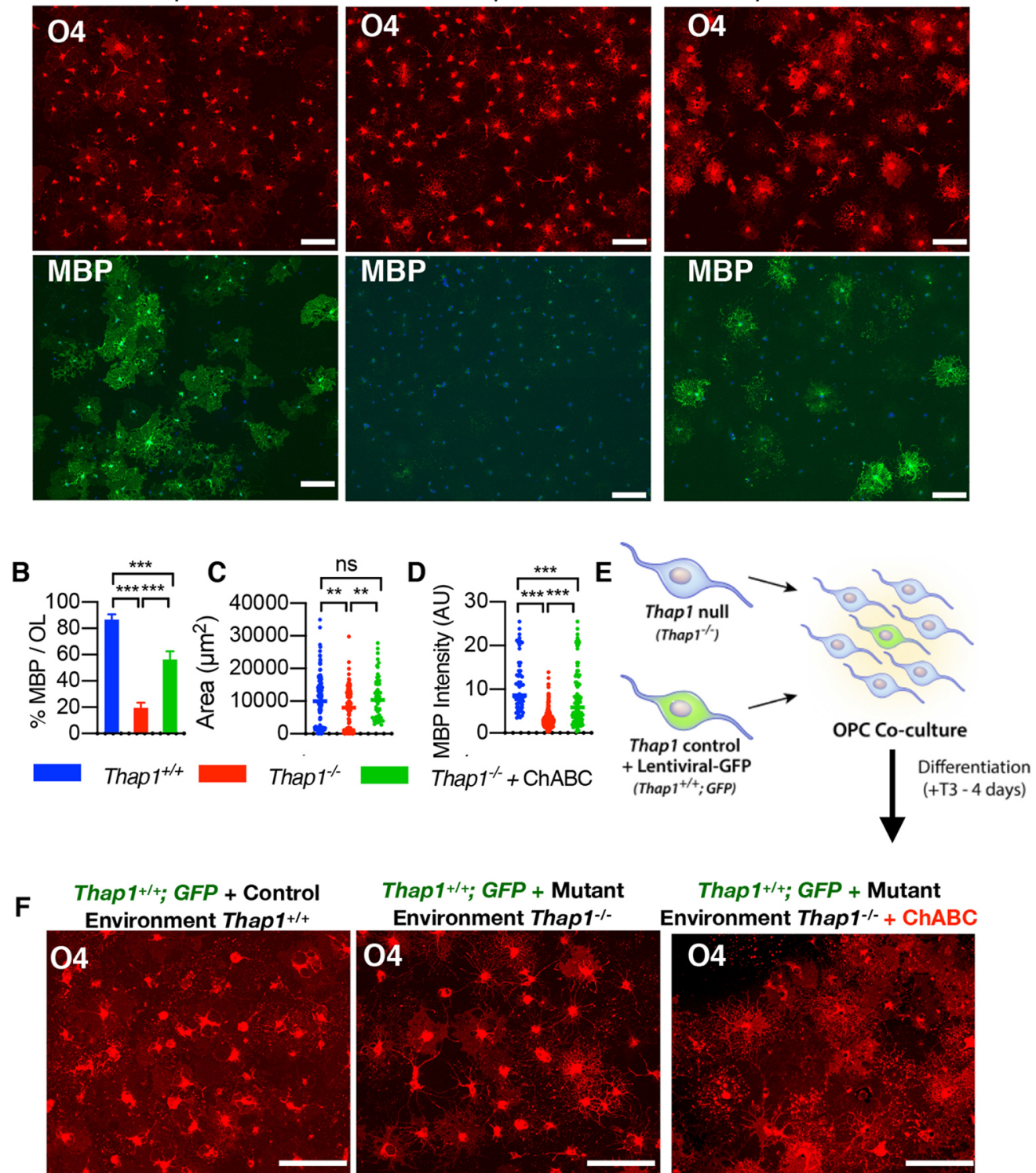

Thap1+/+; GFP + Mutant

Thap 1+/+; GFP + Mutant

Environment Thap1-/-

Environment Thap1 $1-$ + ChABC
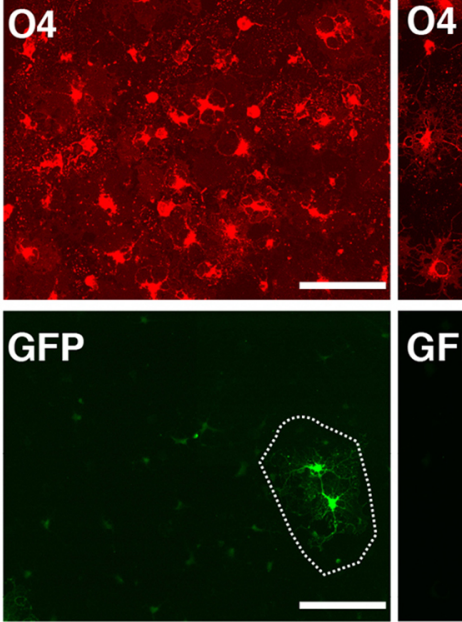

04
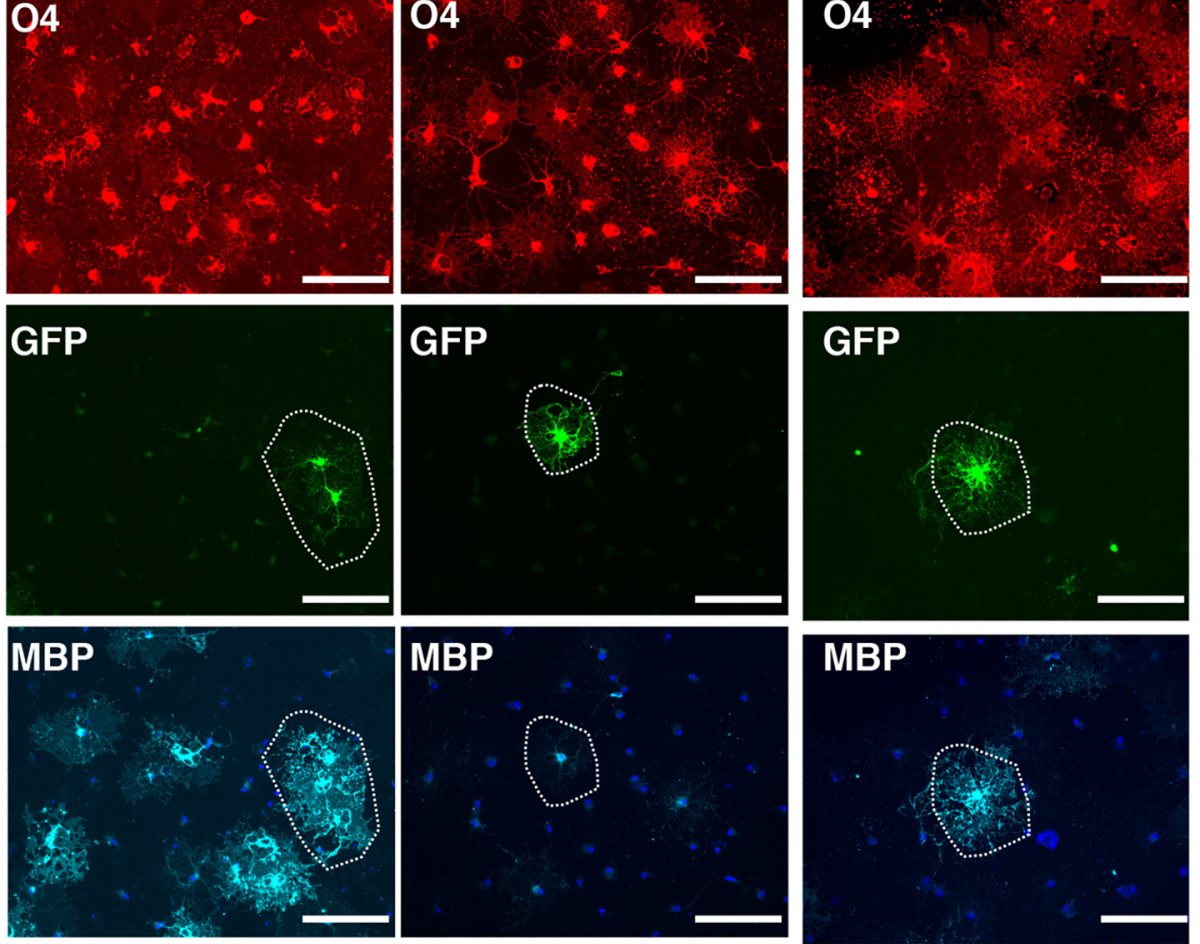

G MBP Intensity - Thap 1+/+; GFP

H OL Area Thap 1+1; ; GFP
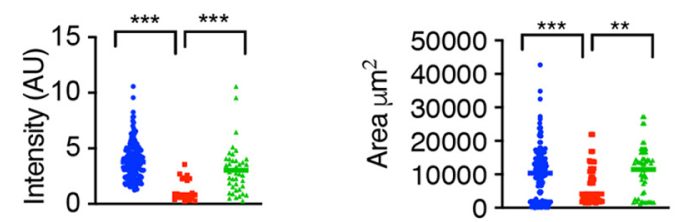

- Control Environment

- Mutant Environment

- Mutant Environment + ChABC

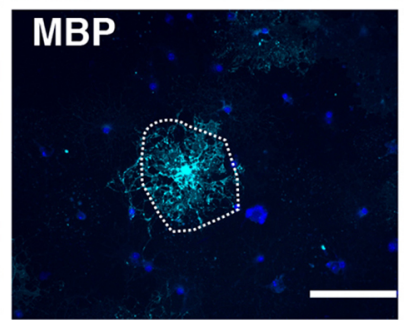


A

GusB

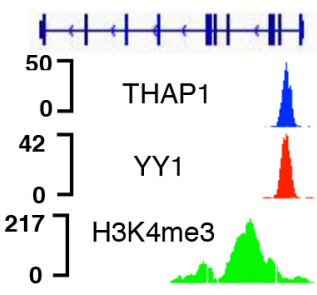

E $\quad$ GusB mRNA

$(\mathrm{OPC}+\mathrm{T} 3)$

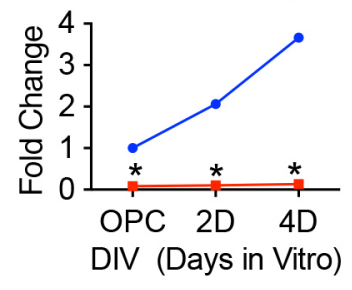

H

GusB mRNA

(Cerebral Cortex)

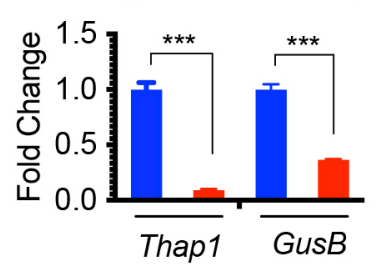

Quantitative chromatin immunoprecipitation (qChIP)

$\begin{array}{lllll}\text { B THAP1 } & \text { C } & \text { YY1 } & \text { D } & \text { H3K4me3 }\end{array}$
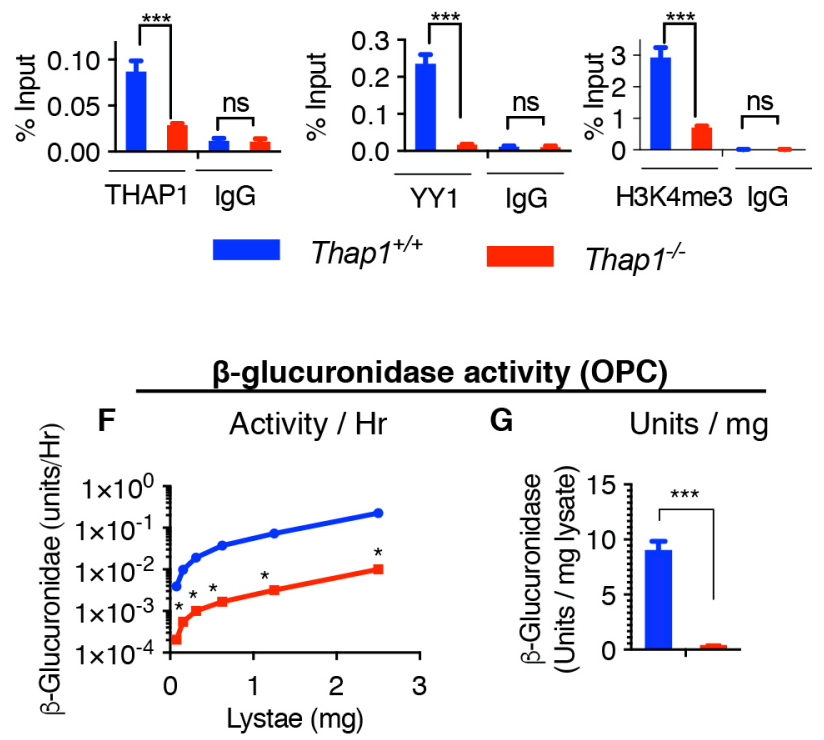
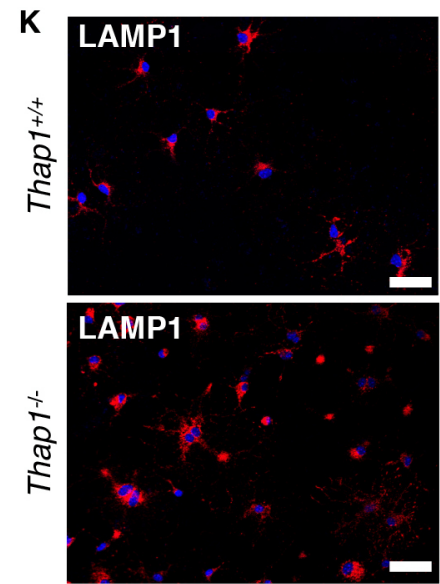

N

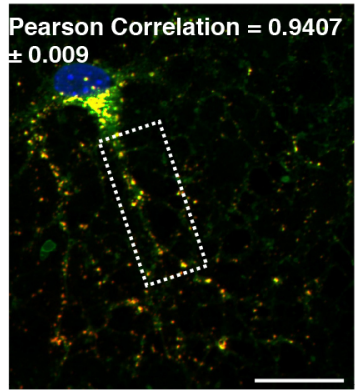

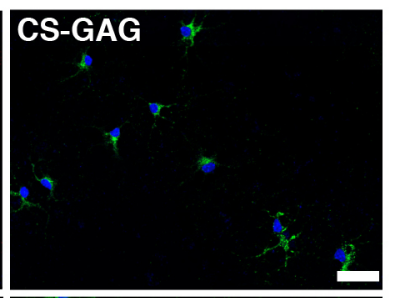
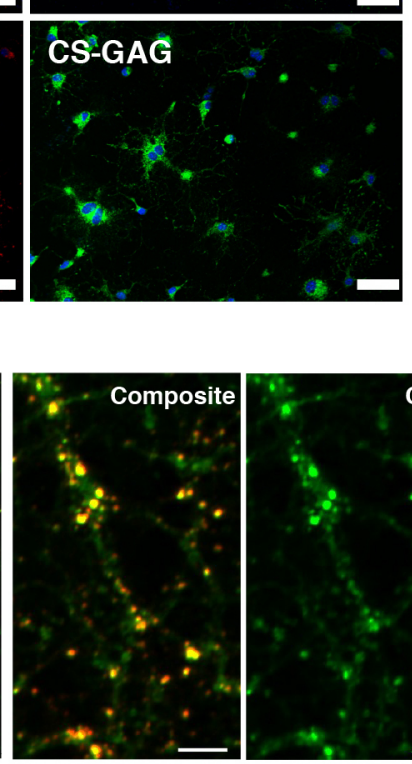
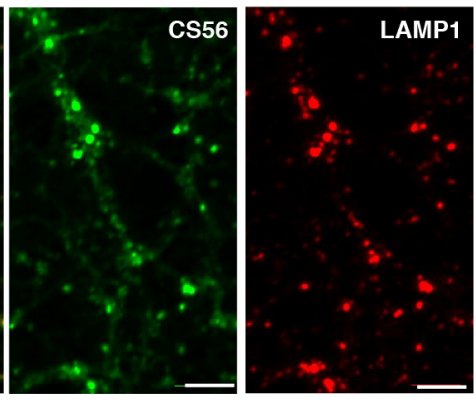
A $\quad \beta$-glucuronidase activity (OPC; TgGUS)

B
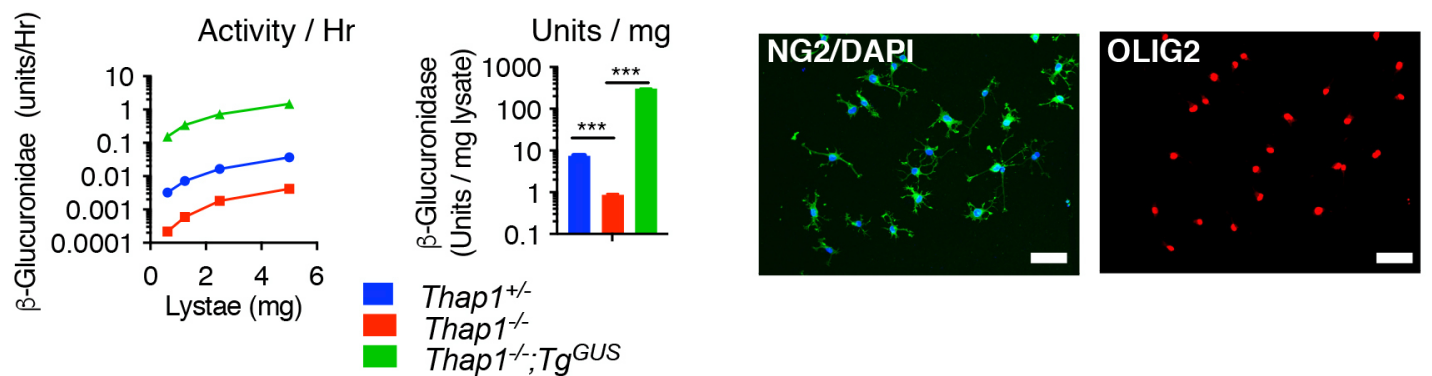

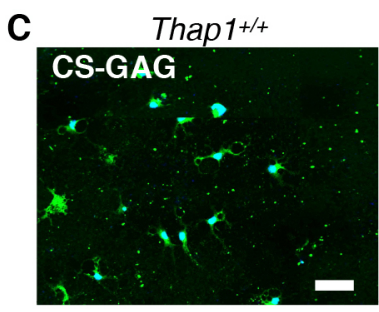

Thap 1-1-

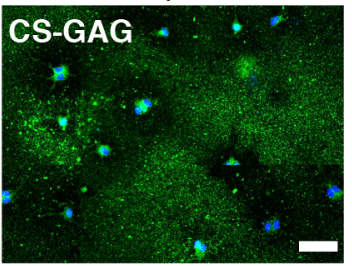

E
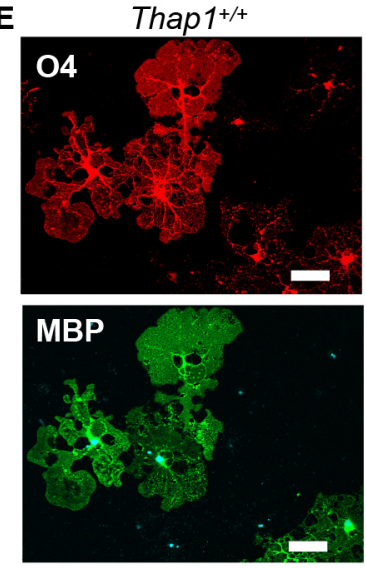

Thap1\%

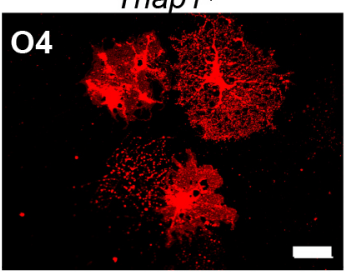

MBP

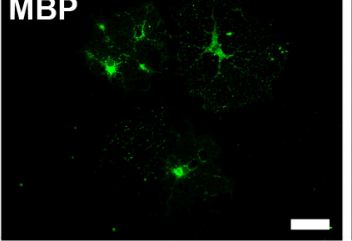

Thap 1- ; TgGus

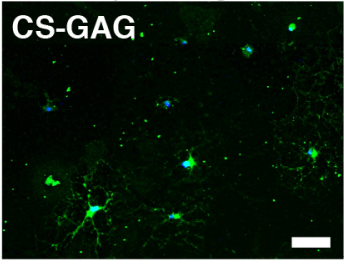

Thap1-1- ; TgGUS
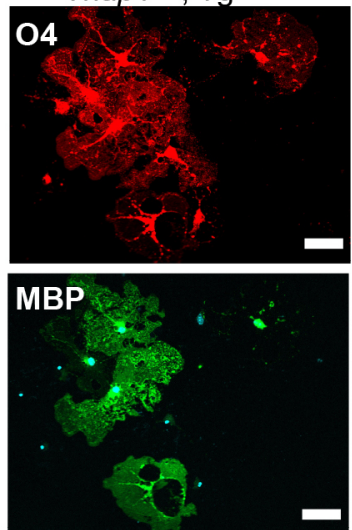

D

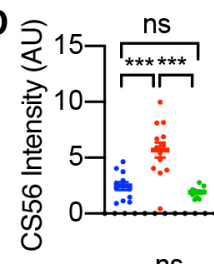

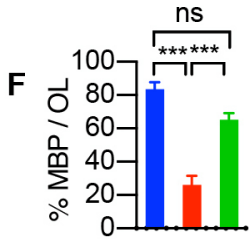

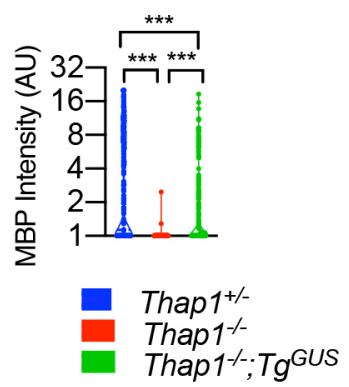


A

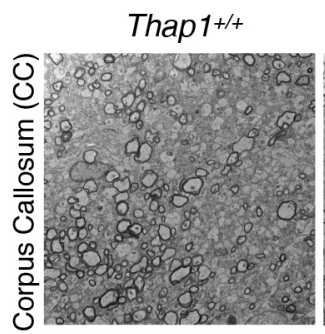

Thap 1+/+-TgGUS

$\mathrm{N}-\mathrm{CKO}$

$\mathrm{N}-\mathrm{CKO} ; \mathrm{Tg}_{\mathrm{GUS}}$

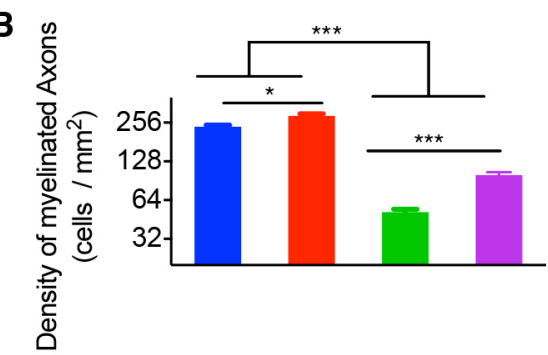

C
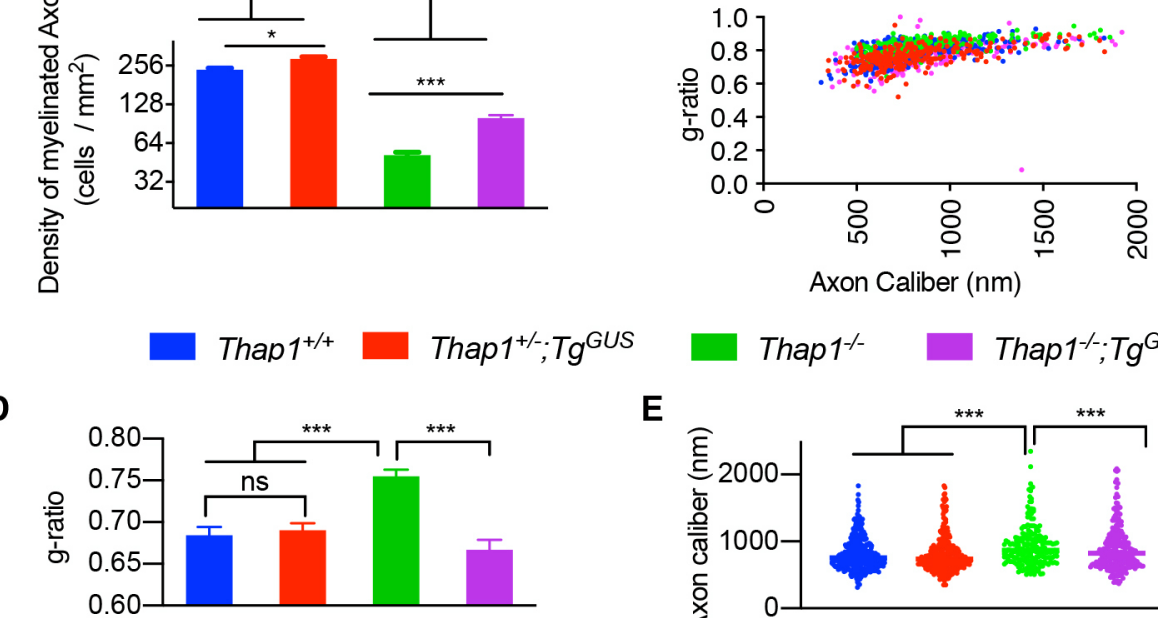

E

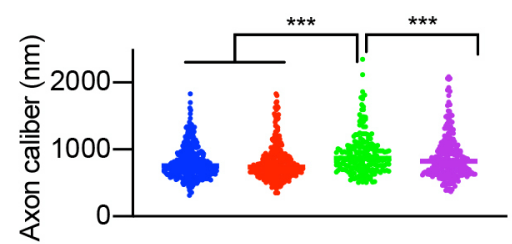

F

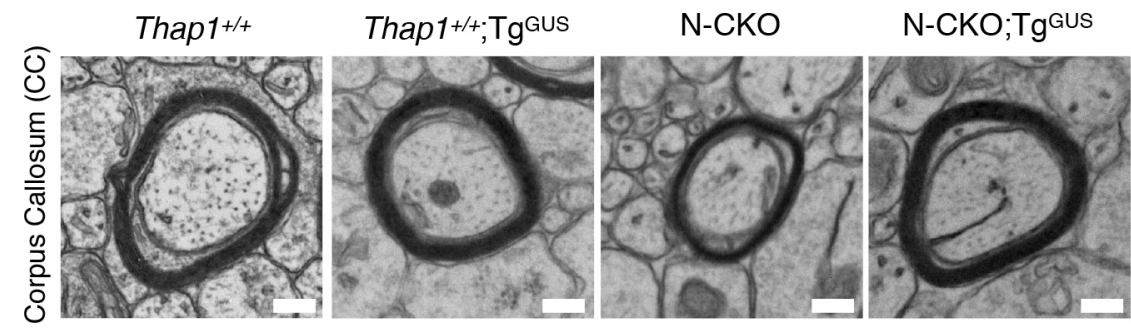

